\title{
Domain wall motion in perpendicular anisotropy nanowires with edge roughness
}

\author{
Maximilian Albert ${ }^{1}$, Matteo Franchin ${ }^{1}$, Thomas Fischbacher ${ }^{1}$, \\ Guido Meier $^{2}$, Hans Fangohr ${ }^{1}$ \\ ${ }^{1}$ School of Engineering Sciences, University of Southampton Southampton, SO17 \\ 1BJ, UK \\ ${ }^{2}$ Institut für Angewandte Physik und Zentrum für Mikrostrukturforschung, \\ Universität Hamburg, Jungiusstrasse 11, 20355 Hamburg, Germany \\ E-mail: fangohr@soton.ac.uk
}

\begin{abstract}
We study field-driven domain wall motion in nanowires with perpendicular magnetic anisotropy using finite-element micromagnetic simulations. Edge roughness is introduced by deforming the finite element mesh, and we vary the correlation length and magnitude of the roughness deformation separately. We observe the Walker breakdown both with and without roughness, with steady domain wall motion for applied fields below the critical Walker field $\mathrm{H}_{\mathrm{c}}$, and oscillatory motion for larger fields. The value of $\mathrm{H}_{\mathrm{c}}$ is not altered in the presence of roughness.

The edge roughness introduces a depinning field. During the transient depinning process from the initial configuration to steady domain wall motion, the domain wall velocity is significantly reduced in comparison to a wire without roughness. The asymptotic domain wall velocity, on the other hand, is virtually unaffected by the roughness, even though the magnetisation reacts to the edge distortions during the entire course of motion, both above and below the Walker breakdown,

A moving domain wall can get pinned again at some later point ('dynamic pinning'). Dynamic pinning is a stochastic process and is observed both for small fields below $\mathrm{H}_{\mathrm{c}}$ as well as for fields of any strength above $\mathrm{H}_{\mathrm{c}}$. In the latter case, where the domain wall shows oscillatory motion and the magnetisation in the domain wall rotates in the film plane, pinning can only occur at positions where the DW reverses direction and the instantaneous velocity is zero, i.e., at the beginning or in the middle of a positional oscillation cycle. In our simulations pinning was only observed at the beginnings of cycles, where the magnetization is pointing along the wire.

The depinning field depends linearly on the magnitude of the edge roughness. The strongest pinning fields are observed for roughness correlation lengths that match the domain wall width.
\end{abstract}

\section{Introduction}

To realise potential storage devices, such as racetrack memory (Parkin et al. 2008), nanowires with low intrinsic pinning as well as nanowires with intended pinning sites for individual domain walls are required. Real nanowires tend to exhibit a roughness at their boundaries which introduces additional complexity. It is reasonable to distinguish 
between the surface roughness originating from the deposition process and the edge roughness that stems from the lithography.

The influence of the latter has been studied for in-plane anisotropy and soft materials (Nakatani et al. 2003), where a vortex-mediated breakdown localised at the sample edges was found and it turned out that the edge roughness can significantly affect the vortex nucleation and annihilation process that determines the behavior of the domain wall. The influence of surface roughness on the domain wall propagation process is also prominent in perpendicular magnetic anisotropy media (PMA) and has been studied, e.g. using the magneto-optical Kerr effect (Metaxas et al. 2007, Kim et al. 2009, Rodríguez-Rodríguez et al. 2010). It has been found that as the wire width decreases, the magnetic domain wall dynamics change from elastic creep in two dimensions to a particle-like stochastic behaviour in one dimension.

Often, pinning sites are desired and can be realised by geometric constrictions to create local confining potentials that act as pinning sites for individual domain walls (Kläui et al. 2005, Hayashi et al. 2006, Im et al. 2009, Bocklage et al. 2009). As an alternative, the local modification of magnetic properties by ion irradiation, e.g. by implanting $\mathrm{Cr}$ ions, is suitable to induce pinning sites (Vogel et al. 2010, Vogel et al. 2011). In this case, a variation of the wire geometry on the nanoscale is not required. The introduction of such magnetic soft spots is attractive due to lower requirements on the lithography in comparison to geometric constrictions on the nanoscale, a smaller distribution of properties due to parallel processing during implantation, and fine tunability of the pinning potential via the chromium ion fluence.

It is important to understand the influence of the usually undesired edge roughness to support work on domain wall propagation in wires without constrictions, with constrictions, and with other intentionally created pinning centres. The role of disorder in in-plane domain wall motion has been studied theoretically, including the effect of edge roughness (Nakatani et al. 2003) and surface roughness (Min et al. 2010) on the domain wall velocity, the interplay of extrinsic pinning with the critical current or field at which the domain wall is depinned and starts to move (Tatara et al. 2006), the influence of thermal excitations and roughness on domain wall motion (Martinez et al. 2007), and domain wall velocity fluctuations due to edge pinning centers (Ryu \& Lee 2009).

Surface roughness has been modelled through introduction of a set of pinning centers for the domain wall (Tatara et al. 2006, Ryu \& Lee 2009). To model edge roughness in the context of finite difference simulations, complete simulation cells have been removed from the micromagnetic sample edge (Martinez et al. 2007), introducing disorder at the length scale of the cell size (of the order of $5 \mathrm{~nm}$ ). Other works use a Voronoi tessellation to model grains in the material, then remove grains from the sample edge, and map this modified sample edge boundary onto the finite-difference grid (Nakatani et al. 2003, Thiaville et al. 2005). The grains are adequately resolved if they are larger than the cell size. To model surface roughness in thin films with finite difference simulations, one can also vary the saturation magnetisation in each finite difference cell instead of varying the height of the cells (Min et al. 2010). The length 


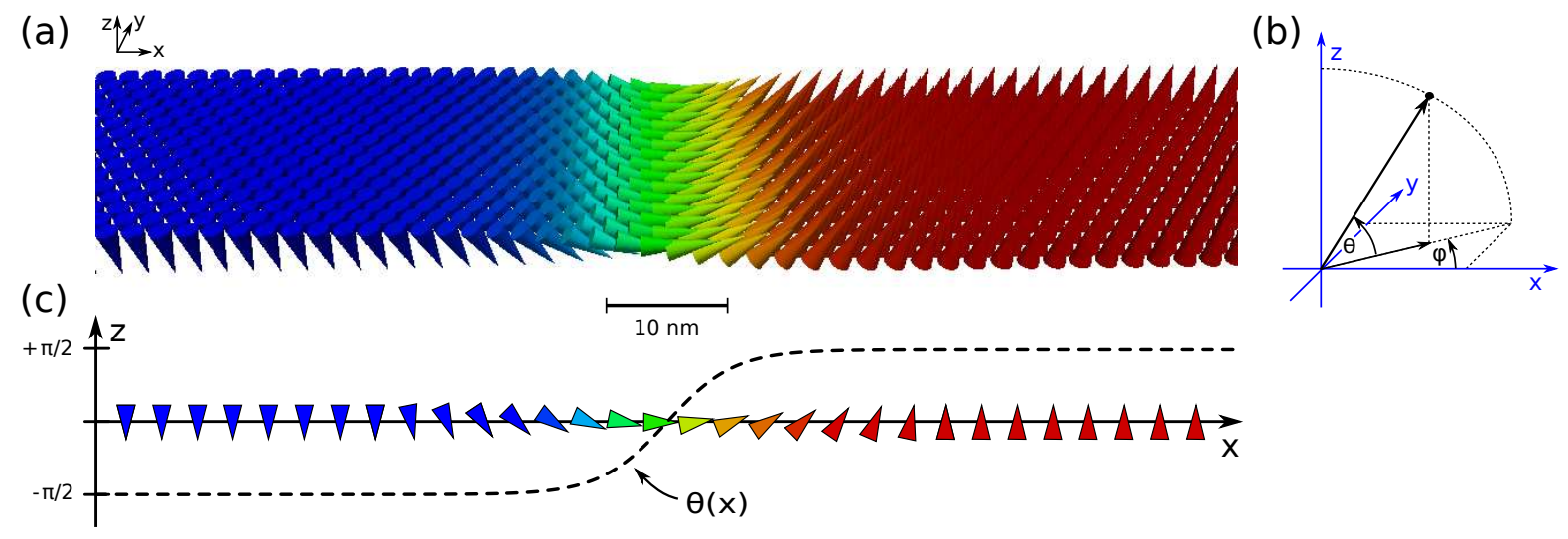

Figure 1. (Color online) (a) Initial Néel wall configuration, after relaxation. (b) Convention of spherical coordinate system. (c) Domain wall profile.

scale of the surface roughness in this model cannot be smaller than the cell size but can be chosen to be longer by varying the saturation magnetisation slowly in space.

In this work, we study the effect of edge roughness which originates from the lithographic sample fabrication process on the domain wall propagation in perpendicular magnetic anisotropy media. A finite-element spatial discretisation of the nanowire and its edge roughness is used. In Sec. 2 we introduce the simulation model, geometry and materials, roughness model and comment on the automated data analysis used. We report simulation results for a smooth nanowire in Sec. 3, before extending the simulation to include edge roughness in Sec. 4. We close with a summary in Sec. 5.

\section{Method}

All simulations are carried out using the micromagnetic simulation package Nmag (Fischbacher et al. 2007, Nmag - a micromagnetic simulation environment 2007) developed at the University of Southampton, which employs a hybrid finite element/boundary element method approach. For our purposes, an advantage over finite difference-based discretisation is that this allows us to model the edge roughness of the system more accurately using a tetrahedral mesh than would be possible with cuboidal cells.

\subsection{Geometry and Material}

The system under consideration is a PMA nanowire with rectangular cross-section and dimensions $1000 \mathrm{~nm} \times 20 \mathrm{~nm} \times 5 \mathrm{~nm}$ in $x, y$ and $z$-axis directions, respectively. The extremal corners have coordinates $(0 \mathrm{~nm}, 0 \mathrm{~nm}, 0 \mathrm{~nm})$ and $(1000 \mathrm{~nm}, 20 \mathrm{~nm}, 5 \mathrm{~nm})$. We have deliberately chosen the wire width to be rather small so that the system can be treated as effectively one-dimensional since the exchange interaction keeps the magnetisation almost constant in $y$ - and $z$-direction. Figure 1 shows the initial configuration for which we set the magnetisation to point down (in negative $z$-direction) 
at the left end of the wire (i.e. at $x=0$ ) and up at the other end, with a domain wall located between the two uniform domains. This two-domain system with domain wall is well described by two parameters: (i) the DW position and (ii) the azimuthal angle $\phi$ of the magnetisation at the DW center. We use spherical coordinates to characterise the orientation of the magnetisation at the centre of the DW, as shown in figure 1 , where $\theta$ is the vertical angle between $\mathbf{M}$ and the $x$-y-plane (polar angle), and $\phi$ is the horizontal angle between $\mathbf{M}$ and the $x$-axis (azimuthal angle). In what follows, we are particularly interested in the value of $\phi$ at the center of the DW, as this - together with the DW position - captures the DW state. The angle $\phi$ will frequently be referred to as the magnetisation angle of the DW.

Inspired by experimental studies (Tanigawa et al. 2009), we use the effective material parameters of a multi-layered $\mathrm{Co} / \mathrm{Ni}$ nanowire with saturation magnetisation $\mathrm{M}_{\mathrm{s}}=6.8 \times 10^{5} \mathrm{~A} / \mathrm{m}$, exchange coupling $A=1.3 \times 10^{-11} \mathrm{~J} / \mathrm{m}$ and uniaxial anisotropy constant $K_{1}=3.8 \times 10^{5} \mathrm{~J} / \mathrm{m}^{3}$. The resulting exchange length is $\sqrt{A / K_{1}}=5.85 \mathrm{~nm}$, the Gilbert damping parameter used is $\alpha=0.032$ (Burrowes et al. 2009).

\subsection{Simulation Stages}

Each simulation consists of two stages: a relaxation phase and the main simulation of the domain wall dynamics. In the relaxation phase we initialise the magnetisation $\mathbf{M}$ in the nanowire to a Néel-like pattern, with $M_{y}=0$ everywhere, i.e., the magnetisation rotates in the $x$-z-plane. The $z$-component $M_{z}$ is initialised to $\tanh \left(\sqrt{K_{1} / A} \cdot(x-200)\right)$, which describes the domain wall pattern for a system when the demagnetising field is neglected, with the DW center located at $x=200 \mathrm{~nm}$. $\neq$ This is then relaxed until it reaches a metastable state as illustrated in figure 1, which takes into account exchange, anisotropy and demagnetisation fields. The relaxation is carried out by numerical integration of the Landau-Lifshitz-Gilbert equation. A large damping coefficient, $\alpha=1.0$, is used to speed up the relaxation. The relaxed systems exhibits a Néel domain wall, as shown in figure 1, to avoid surface charges on the edges which would be associated with a Bloch wall.

Once equilibrium is reached the second stage is started. The damping is set to the realistic value, $\alpha=0.032$, and a constant external field $H_{\text {ext }}$ is applied along the $z$-axis. The response of the magnetisation is then computed for $20 \mathrm{~ns}$. Every $0.1 \mathrm{~ns}$ the domain wall position (along the $x$-axis) is computed by finding the zero-crossing of $\theta$ along the nanowire axis. This is done by probing $\theta$ at 2000 auxiliary nodes along this line and determining the two adjacent nodes where a sign change occurs. Then $\theta$ is interpolated linearly between these adjacent nodes to determine the position of the zero-crossing. $\S$

$\ddagger$ Control simulations suggest that boundary effects are negligible further than $\approx 150 \mathrm{~nm}$ away from the ends of the nanowire. In this work no data of domain walls outside this range was used.

$\S$ The determination of the zero-crossing uses a Python function which is given to the simulation framework with the instruction to call it every $0.1 \mathrm{~ns}$ during the computation, which avoids storing all the field data for later postprocessing. The integration of the micromagnetic simulation tool into an existing programming language thus simplifies data capture and analysis here (Fischbacher et al. 2009). 
(a)
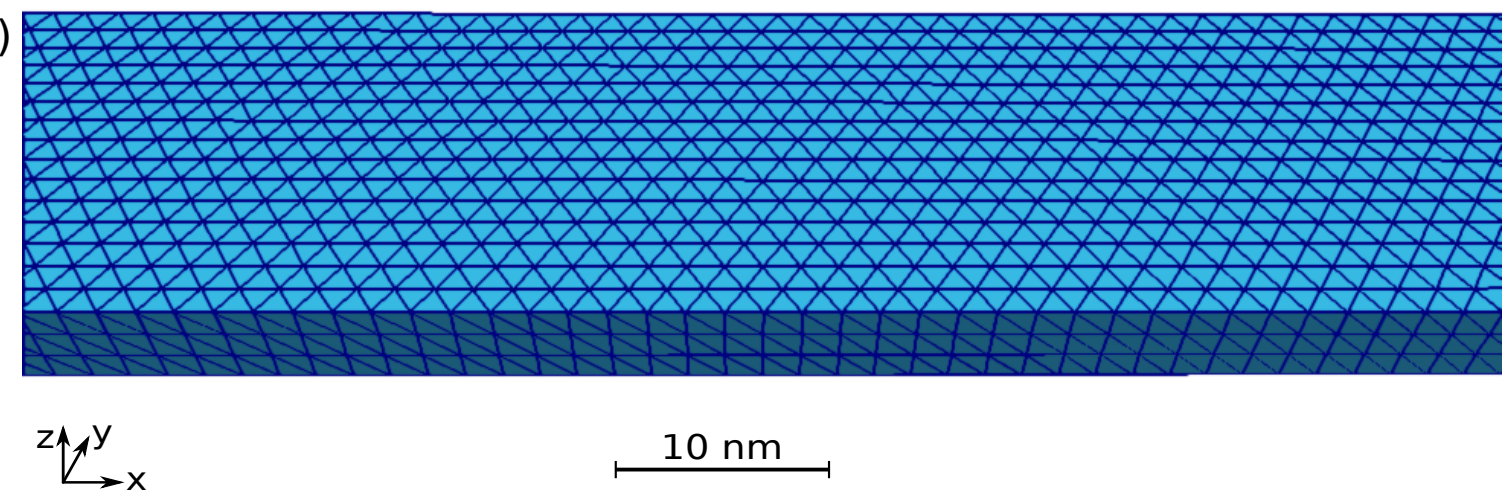

(b)

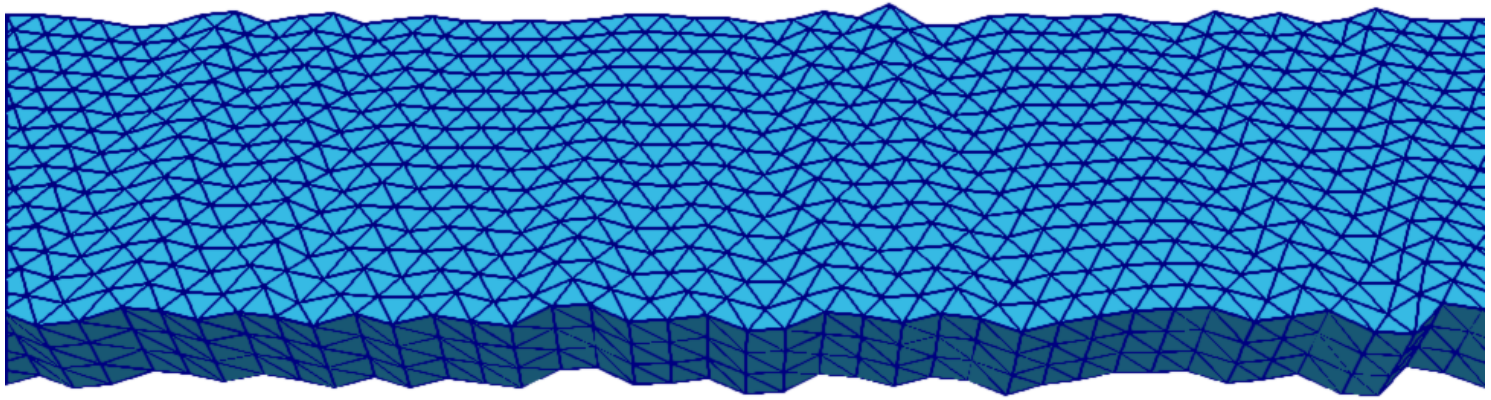

Figure 2. (Color online) (a) Smooth mesh. For this mesh there are three layers of tetrahedra in $z$-direction representing the film thickness of $5 \mathrm{~nm}$ and 15 layers in $y$-direction extending $20 \mathrm{~nm}$. Only a part of the mesh of the wire is shown in the $x$-direction. (b) Rough version of the same mesh (with correlation length $c=2 \mathrm{~nm}$ and distortion amplitude $d=0.4 \mathrm{~nm}$ ).

Moreover, at the position of the DW center the magnetisation angle $\phi$ is recorded and both the domain wall position and magnetisation angle, together with the current time step, are written to an output file for subsequent analysis.

\subsection{Roughness Model}

In this work, we use a finite element-based discretisation of space. This allows to model rough edges explicitly using a distorted finite element mesh. We start from a smooth tetrahedral mesh as shown in figure 2 (a) with dimensions $1000 \mathrm{~nm} \times 20 \mathrm{~nm} \times 5 \mathrm{~nm}$, which is subsequently distorted at front and rear edges as shown in figure 2 (b). In the following, the term edge or edge surface always refers to the two surfaces of dimensions $1000 \mathrm{~nm} \times 5 \mathrm{~nm}$ at the the long edges of the wire parallel to the $x-z$ plane.

The overall distortion process works as follows. We first construct a 'distortion function' $f(x)$. This function specifies the amount by which each node lying on the front edge surface of the mesh (where $y=0 \mathrm{~nm}$ ) is displaced in $y$-direction, as a function of the $x$-coordinate of the node. Analogously, the nodes at the rear edge surface (where $y=20 \mathrm{~nm}$ ) are displaced using a second, independently constructed distortion function $g(x)$ so that both edges of the mesh are distorted differently. The positions of the 
internal mesh nodes are then rescaled in order to fit between the new distorted sides.

The distortion functions $f$ and $g$ are constructed as follows. First we pick equidistant positions $x_{i}$ along the $x$-axis. These are just auxiliary entities and completely independent of the actual mesh nodes. The distance between two neighbouring auxiliary nodes is referred to as the correlation length $c$ of the distortion. Next, random values $f\left(x_{i}\right)$ and $g\left(x_{i}\right)$ are assigned to each position $x_{i}$, chosen from a Gaussian distribution with mean 0 and standard deviation $d$, which is referred to as the distortion amplitude of the roughness or simply as the roughness level. Finally, the random values $f\left(x_{i}\right)$ and $g\left(x_{i}\right)$ are interpolated smoothly to obtain the continuous distortion functions $f(x)$ and $g(x)$. The whole process is illustrated in figure 3. In order to make the randomisation reproducible, it is possible to specify a seed for the internal random number generator. This allows us for a given $c$ to produce meshes with the same 'shape of roughness' but different roughness amplitudes: the distortion functions of these meshes are just scaled versions of each other. We introduce the distortion functions $f$ and $g$, rather than displaceing the mesh nodes on the edges randomly and thus independently of each other, in order to be able to control the roughness correlation length independently of the actual mesh discretization (assuming that the edge length of the tetrahedra for the chosen mesh discretisation is smaller than the correlation length $c$ ).

The effective roughness length scale, which we define as the average width of the peaks and troughs of the rough edge, or - equivalently - the distance between adjacent local minima in $f(x)$ and $g(x)$, is larger than the correlation length $c$ as visible in figure $3(\mathrm{~b})$. Statistical analysis of this effective roughness length scale for a range of disorder functions $f(x)$ and $g(x)$ shows that the effective roughness length is given by $\approx 2.76 c$ (see figure $3(\mathrm{~d}))$.

There are several conceivable ways of modelling edge roughness, including locally varying material parameters or Voronoi cell approaches, as mentioned in Sec. 1. The method used here models the kind of roughness associated with irregularities in the sample geometry originating from electron beam lithography, due to the remaining jitter of the electron beam around a zero position. The amount of this jitter is modelled by the roughness amplitude $d$. Depending on the speed of the beam along the edge this jitter is 'spread out' over a certain distance, which is modelled by the correlation length $c$. We note, however, that there is a second source of roughness due to the chemical process of transferring the latent image of the nanowire after exposure, which involves rather long-chain molecules. Unless special care is taken this is usually the dominant source of roughness.

\subsection{Data Analysis}

We have carried out over 24,000 simulations where we systematically vary the external field strength $H$, roughness correlation length $c$, and roughness amplitude $d$. For each configuration, i.e. combination of the three parameters $(H, c, d)$, we carry out one simulation run. Each simulation run produces an output file containing data recorded 
(a)

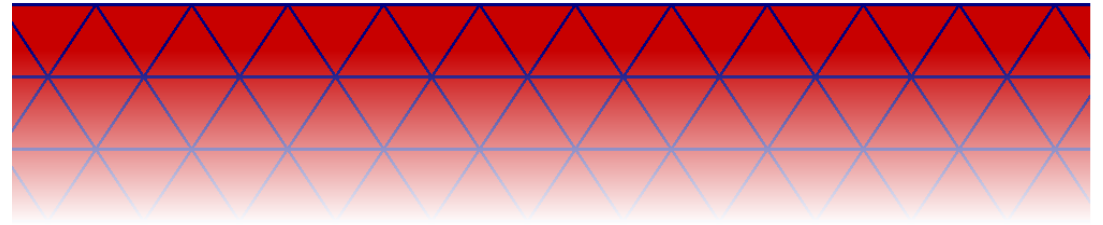

(b)

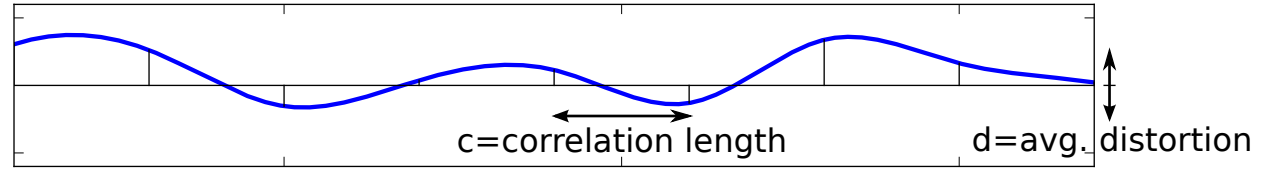

(c)

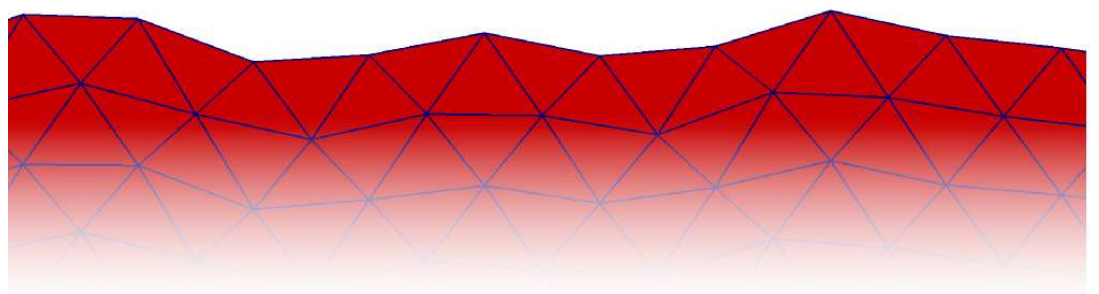

$(d)$

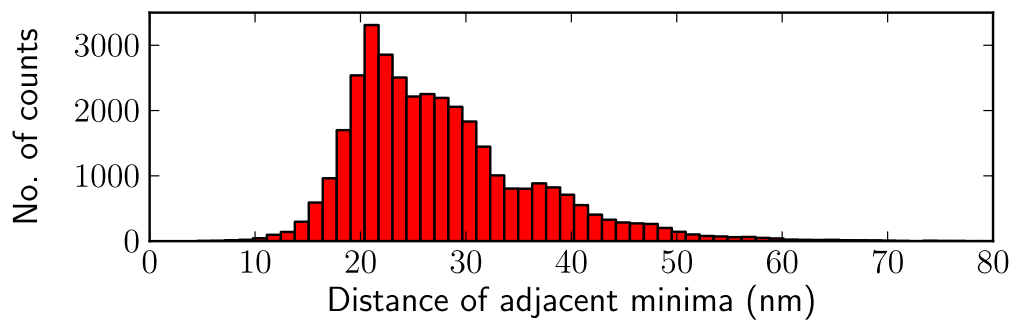

Figure 3. (Color online) Illustration of the edge distortion process, showing a top view of the rear edge of the nanowire. (a) Original mesh. (b) Construction of the distortion function $g$ with certain correlation length and distortion amplitude. (c) The mesh after distortion with $g$. The contour of the distorted mesh follows the outline of $g$. (d) Distribution of the distances between adjacent local minima. The data was gathered from a collection of 1000 different distortion functions, each produced with a different randomisation seed at the fixed correlation length $c=10 \mathrm{~nm}$. The mean distance is $27.6 \mathrm{~nm}=2.76 \times c$, which defines the effective roughness length scale.

every $0.1 \mathrm{~ns}$ between $0 \mathrm{~ns}$ and $20 \mathrm{~ns}$. For each of the time steps the corresponding computed domain wall position and the magnetisation angle $\phi$ inside the domain wall are recorded.

The main observable we are interested in for each configuration is the DW velocity $v_{\mathrm{x}}(t)$, which is derived from the DW position $r_{\mathrm{x}}(t)$ as a function of time. All other quantities, such as the depinning field for a given roughness level, can be computed from this. Our simulation results of a smooth nanowire in Sec. 3 and a nanowire with edge roughness in Sec. 4 agree qualitatively with Walker's prediction (Schryer \& Walker 1974) that there is steady domain wall motion for applied fields $H$ below a critical field $\mathrm{H}_{\mathrm{c}}$, and oscillatory motion for larger applied fields. We need to distinguish between these two regimes to apply appropriate methods to compute the domain wall velocity. To do this, we use the angle $\phi$ as the criterion: in the steady-motion regime the magnetisation 
angle $\phi$ approaches an asymptotic value $|\phi|<\frac{\pi}{2}$, whereas in the oscillatory regime the magnetisation inside the DW keeps precessing indefinitely, so that $|\phi|$ grows to infinity.

2.4.1. Mean velocity The simplest way to compute the domain wall velocity is to subtract the initial domain wall position $r_{\mathrm{x}}\left(t_{0}\right)$ at time $t_{0}$ from the final position $r_{\mathrm{x}}\left(t_{\mathrm{f}}\right)$ at time $t_{\mathrm{f}}$, and to divide by the time it took to travel that distance:

$$
v_{\mathrm{x}}^{\text {mean }}=\frac{r_{\mathrm{x}}\left(t_{\mathrm{f}}\right)-r_{\mathrm{x}}\left(t_{0}\right)}{t_{\mathrm{f}}-t_{0}}
$$

Later analysis in Sections 3 and 4 shows, however, that the initial depinning process of a domain wall is qualitatively different from the subsequent domain wall motion: during the depinning process, the domain wall increases its velocity from zero to an asymptotic value for fields below the Walker breakdown, or asymptotic periodic behaviour for fields above the Walker breakdown. As this increase does not happen instantaneously, the transient process will reduce the mean velocity in the steady-motion regime if computed using (1). The decrease will depend on the simulated time: the longer we run the simulation, the smaller the reduction of $v_{\mathrm{x}}^{\text {mean }}$ due to the initial transient, and the less do mean and asymptotic velocities differ. Moreover, for oscillatory motion there can be some variation in the mean velocity as computed using (1) depending on how much of the last oscillation cycle is completed when the simulation exits.

2.4.2. Asymptotic velocity We thus use another method to compute the domain wall velocity, which eliminates the reduction of $v_{\mathrm{x}}^{\text {mean }}$ due to the initial transient and removes artefacts due to incomplete oscillation cycles. To distinguish from the mean velocity, we refer to this as the asymptotic velocity.

In the oscillatory case we identify the time and position of the DW at the beginning of each oscillation cycle, i.e. when the angle $\phi$ is a multiple of $\pi$. Using a least-squares approximation we then fit a line through these position as a function of time, and compute the velocity as the slope of the fitted line as shown in figure 4 (a).|| The initial depinning transient is negligible for external fields above $\mathrm{H}_{\mathrm{c}}$ and therefore does not affect the calculation of the asymptotic velocity in this regime.

For applied fields below the Walker breakdown, the domain wall shows nonoscillatory motion. To eliminate the initial transient from the data analysis in this regime, we identify the first time $t_{1}$ and domain wall position $r_{\mathrm{x}}\left(t_{1}\right)$ from which onwards the plot of domain wall position as function of time shows an approximately linear dependence. We then compute the velocity as

$$
v_{\mathrm{x}}^{\text {asymptotic }}=\frac{r_{\mathrm{x}}\left(t_{\mathrm{f}}\right)-r_{\mathrm{x}}\left(t_{1}\right)}{t_{\mathrm{f}}-t_{1}}
$$

\| Contrary to the usual convention, in this paper we plot time $t$ along the vertical axis and the DW position $x$ along the horizontal axis. This is consistent with the orientation of the nanowire in figures 1 , 2,7 , and allows better comparison between plots in the same figure. With this convention the velocities of the domain walls are actually given by the inverse slope of the trajectories in these figures. Hence a steeper line indicates a slower domain wall since it moves less far along the $x$-axis during the course of the simulation. 
(a)

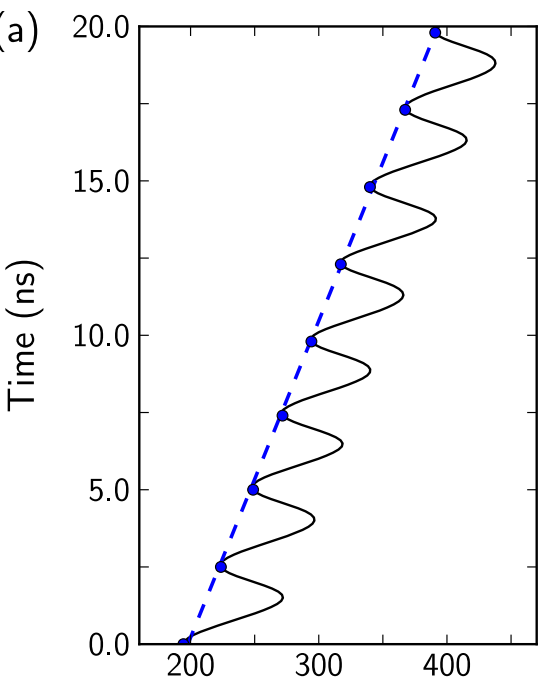

(b)

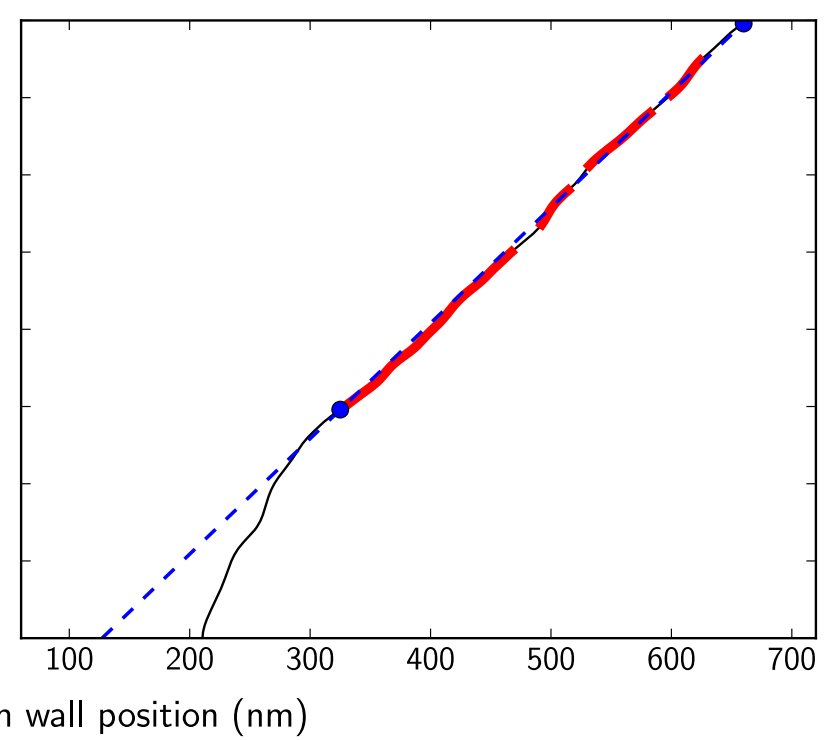

Figure 4. (Color online) Illustration of the computation of the asymptotic domain wall velocity. (a) In the oscillatory case, the positions and times for the beginning of each oscillation cycle are identified, and a line is fitted through them (dashed blue line). The line's slope provides the velocity. (b) In the steady-motion regime below the Walker breakdown field, we identify the beginning of the first straight segment in the plot (thickened red part), and take the position and time of this point, together with the last position and time, to compute the asymptotic velocity. The interpolated points for both cases are marked with blue dots in both plots.

We identify segments of the $r_{\mathrm{x}}(t)$ function where the DW exhibits approximately straight motion by using a Savitzky-Golay filter (Press et al. 1992, Ch. 14.8). This computes for each recorded time step the second derivative of a smoothed version of the trajectory, which gives an indication of the curvature of the trajectory at that point. By discarding points where the second derivatives are above a given threshold we identify one or more segments where the DW exhibits approximately straight motion as shown in figure 4 (b) (segments marked with thick red lines).

2.4.3. Pinning Using these tools, we can also identify when a domain wall (i) is never depinned, or (ii) gets pinned again after having moved a certain distance (dynamic pinning). In case (i), we record the domain wall velocity as zero for both $v_{\mathrm{x}}^{\text {mean }}$ and $v_{\mathrm{x}}^{\text {asymptotic }}$. In case (ii), we compute the mean velocity $v_{\mathrm{x}}^{\text {mean }}$ using (1) but record no velocity for $v_{\mathrm{x}}^{\text {asymptotic }}$ as the motion is too irregular to estimate the asymptotic velocity.

\section{Smooth Nanowire}

In the following we give a summary of the dynamics of perpendicular domain walls in a smooth nanowire in external fields. These results present the reference for the rough nanowire studied in section 4 . 

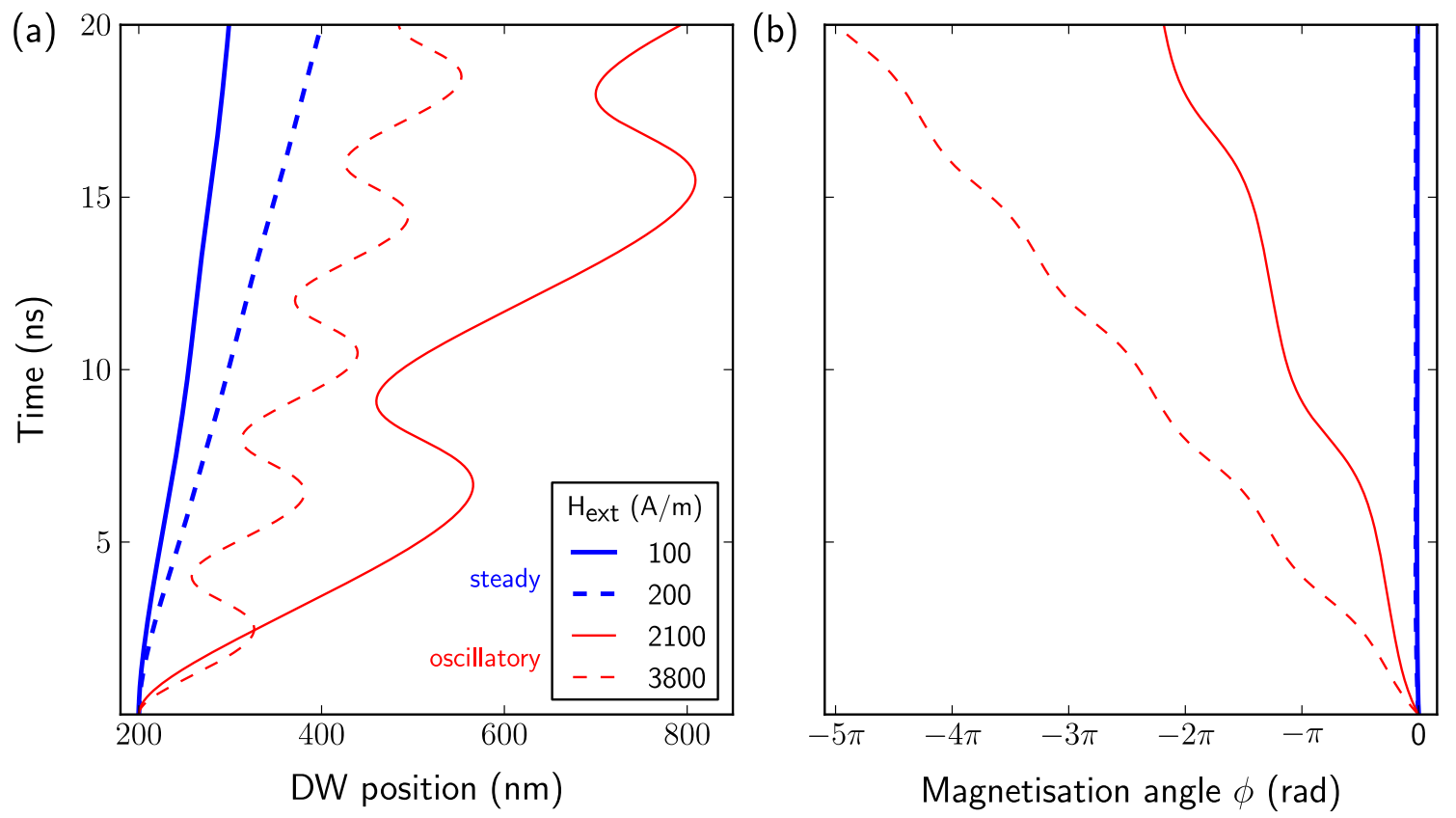

Figure 5. (Color online) (a) Sample DW trajectories for different applied fields $H$ : steady (thick blue lines) and oscillatory (thin red lines). (b) The corresponding magnetisation angles $\phi$.

The external field $\mathrm{H}_{\text {ext }}$ is applied along the negative out-of-plane $z$-direction (see Figure 1). The domain wall then moves to the right, i.e. along the positive $x$-direction. This behaviour can be understood in terms of energy minimisation: the domain wall moves to the right to allow the left domain to grow. Indeed, a wider left domain corresponds to an "increased" alignment of the magnetisation to the applied field and a reduction of the Zeeman and total energy.

Figure 5 (a) shows the position of the domain wall against time for four different applied field strengths. We see that the domain wall starts to move as the field is applied, in all the considered cases. For the two lower fields (blue thick lines), the motion is 'steady', with velocity increasing with the applied field. In contrast, for the two higher applied fields (thin red lines) the motion of the domain wall is oscillatory. The domain wall moves backwards and forwards as a function of time, but moves further forward than backward in each cycle, leading to a net positive velocity. The frequency of the oscillation depends on the external field strength, and is higher for the larger external field (dashed thin line), while the net velocity is lower for the larger external field.

Figure $5(\mathrm{~b})$ shows the magnetisation angle at the DW centre, $\phi$, against time for the same simulations considered in figure 5 (a). For the steady motion curves (thick lines), $\phi$ changes from zero at time $t=0$ to a very small negative value and then remains constant. In the oscillatory regime (thin lines), $\phi$ behaves differently: it grows continuously as a function of time, and grows the faster the higher the applied field is. For the steady-motion curves considered in figure 5 the angle is small because the fields 


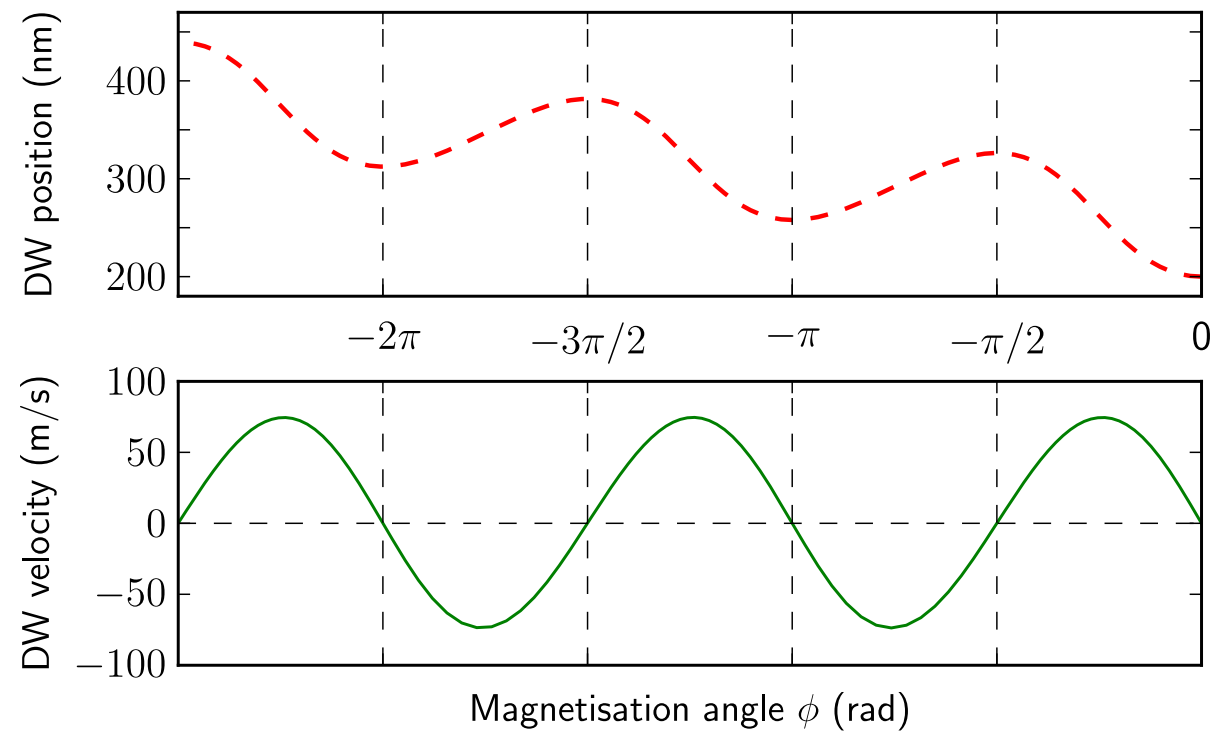

Figure 6. Domain wall position (top) and domain wall velocity (bottom) as a function of magnetisation angle $\phi$ for $H=3.8 \mathrm{kA} / \mathrm{m}$ in the oscillatory regime. The figure should be read 'from right to left' because the angle $\phi$ increases in the negative direction during the DW motion.

are weak; for fields close to $\mathrm{H}_{\mathrm{c}}$ the asymptotic angle can become quite large until at $\mathrm{H}_{\mathrm{c}}$ it reaches $-\frac{\pi}{2}$ and 'tips over' so that it can keep growing grow continuously.

Figure 6 combines the data from the two previous plots and shows domain wall position (top) and velocity (bottom) as a function of the magnetisation angle $\phi$ for the $H=3.8 \mathrm{kA} / \mathrm{m}$ curve in figure 5 . The figure should be read 'from right to left' because the angle $\phi$ increases in the negative direction during motion. We can see that the domain wall position increases as $\phi$ grows from 0 to $-\frac{\pi}{2}$ and that the velocity remains positive in this interval. From $\phi=-\frac{\pi}{2}$ the domain wall moves backwards until $\phi=-\pi$, at which point the cycle repeats. We note that $\phi$ changes by $-\pi$ (not $-2 \pi$ ) while the domain wall position completes an oscillation cycle, so that the magnetisation in the domain wall centre points in the negative $x$ direction at the end of the positional cycle (i.e. $\phi=-\pi$ ) whereas it was pointing in the positive $x$ direction at the beginning. After one more positional cycle the angle also returns to the original position, so that the DW position completes two cycles during a full rotation of the magnetisation angle.

Figure 7 shows a set of corresponding magnetisation configurations for different magnetisation angles $\phi$. We use these figures to interpret the oscillatory motion. Starting from figure 7 (a), the applied external field forces the magnetisation to precess so that $\phi$ changes from 0 to a negative value. The configuration in figure 7 (a), resembles a Néel wall which has no surface charges on the front and back edge of the wire. However, once the magnetisation in the domain wall starts to rotate in the $x$ - $y$ plane, surface charges start to appear on the sides of the wire as in figure 7 (b). These increase the demagnetisation energy of the system, and energy has to be found to allow this change. This energy is provided through the Zeeman term by growing the left domain in the 
(a)

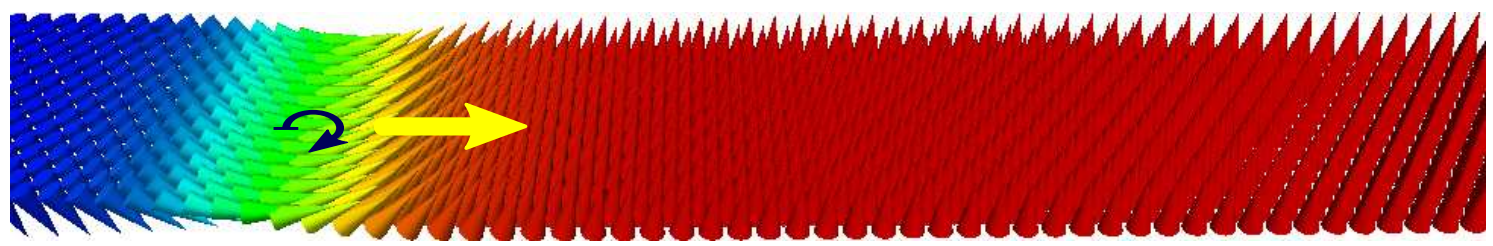

(b)

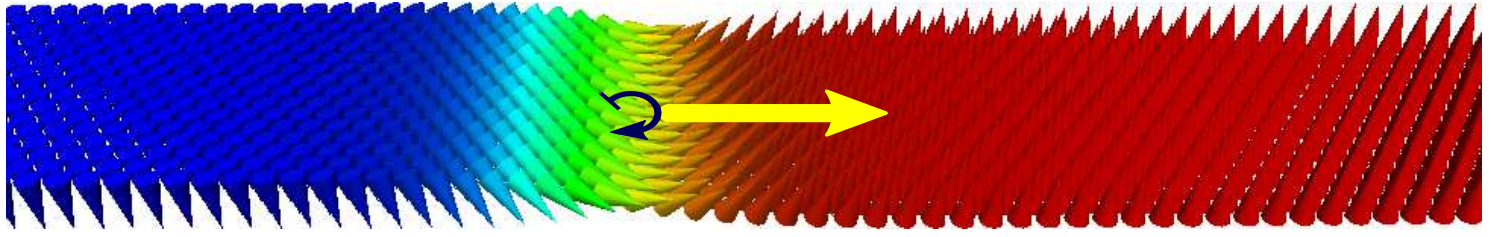

(c)

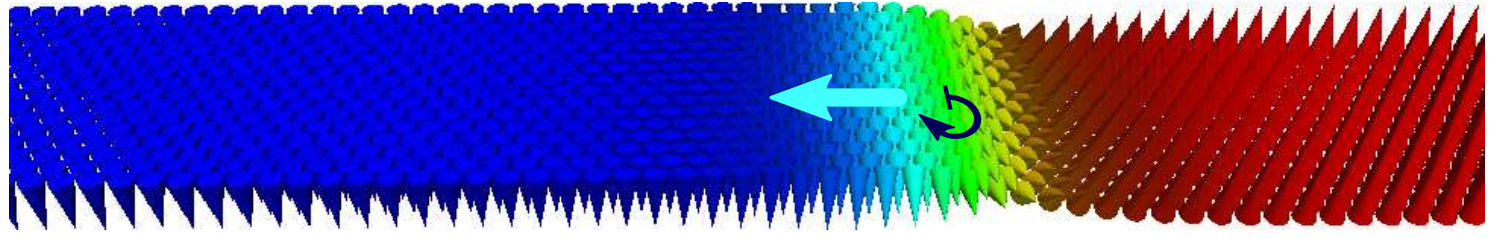

(d)

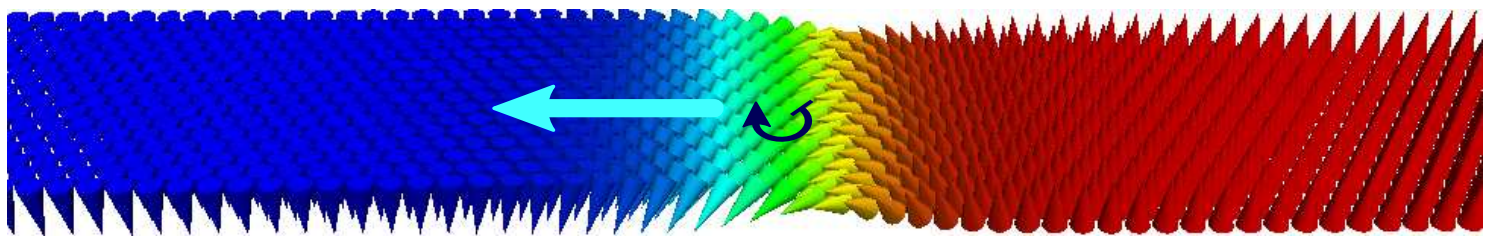

(e)

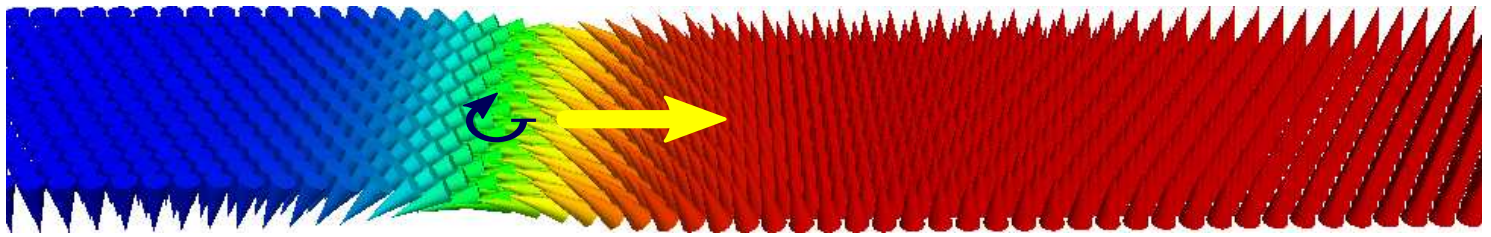

$\stackrel{\mathrm{z} y^{\mathrm{y}}}{\longrightarrow}$

$10 \mathrm{~nm}$

Figure 7. (Color online) Snapshots of a domain wall moving in a smooth nanowire $\left(\mathrm{H}_{\text {ext }}=9 \mathrm{kA} / \mathrm{m}>\mathrm{H}_{\mathrm{c}}\right)$. The pictures show half a rotation of the first oscillation, with time increasing from top to bottom. (a) The initial configuration, cf. figure 1. (b) The domain wall moves to the right as the magnetisation angle $\phi$ precesses around the vertical axis. (c) At $\phi=\frac{\pi}{2}$ the DW reverses direction and (d) starts moving to the left. (e) At $\phi=\pi$ another reversal of direction occurs and the DW moves to the right again. The same procedure repeats itself with two more direction reversals at $\phi=3 / 2 \pi$ and $\phi=2 \pi$ (both not shown) until the angle $\phi$ has completed a full turn and the DW starts its third oscillation. Large superimposed arrows indicate velocity of the domain wall. The thin curly arrows indicate the direction of rotation of the magnetic moments in the domain wall. 
wire: both magnetisation and applied field point in the negative $z$ direction in this domain. Growing the domain on the left means that the domain wall needs to move to the right, i.e. towards larger $x$ values. The further $\phi$ grows towards $-\frac{\pi}{2}$, the further the domain wall needs to move.

For weak applied fields, the growing demagnetisation field can counteract the precession torque from the applied field and the system settles into a steady state with fixed angle $\phi$ in which the domain wall moves continuously towards larger $x$-values (thick lines in figure 5). For large applied fields, however, $\phi$ eventually reaches $-\frac{\pi}{2}$, corresponding to snapshot 7 (c) where the magnetisation is pointing in the negative $y$ direction, which is best visible in the centre of the domain wall. While $\phi$ increases further from $-\frac{\pi}{2}$ to $-\pi$, the surface charges reduce and thus the demagnetisation energy is reduced. This is compensated by the domain wall moving back to the left to shrink the domain on the left that is aligned with the applied field, as shown in figure 7 (d). When $\phi$ reaches $-\pi$, the surface charges have disappeared and the cycle will start again in a mirror-symmetric way, explaining why the domain wall moves backwards and forwards twice while $\phi$ increases from 0 to $-2 \pi$.

If there was no damping in this system (i.e. $\alpha=0$ ), the domain wall would move back to its starting position when $\phi$ reaches multiples of $\pi$. It is the damping term that allows to release energy from the system, and this results in a net motion of the domain wall in the positive $x$ direction due to the applied field in the negative $z$ direction. We also note that if the simulations are carried out without consideration of the demagnetising field, then the oscillations in the domain wall position cannot be observed.

We thus find two different domain wall motion regimes: steady motion for applied fields $H$ below the so-called Walker breakdown field $\mathrm{H}_{\mathrm{c}}$ and oscillatory motion for $H>\mathrm{H}_{\mathrm{c}}$ (Schryer \& Walker 1974). Figure 8 shows the DW velocity as a function of the external field. In a smooth system as simulated here, i.e. in the absence of any domain wall pinning due to roughness, the domain wall starts to move if any non-zero external field is applied. In line with Walker's prediction the domain wall velocity increases with the applied field up to the Walker breakdown field $\mathrm{H}_{\mathrm{c}}$ at which the velocity reaches its maximum. For larger applied fields, the domain wall velocity decreases. Sample simulations with field strengths much larger than $\mathrm{H}_{c}$ have shown that the DW velocity assumes a minimum around $H=30 \mathrm{kA} / \mathrm{m}$ and increases again for even stronger external fields.

\section{Nanowire with edge roughness}

In this section we introduce edge roughness to the wire geometry and repeat the simulations of field driven domain wall motion from section 3 in the presence of this disorder. The relaxation stage of the simulations (section 2.2) allows the domain wall to settle into an energetically favourable position that takes the edge roughness into account, which may thus deviate slightly from the position in the smooth case. As a 


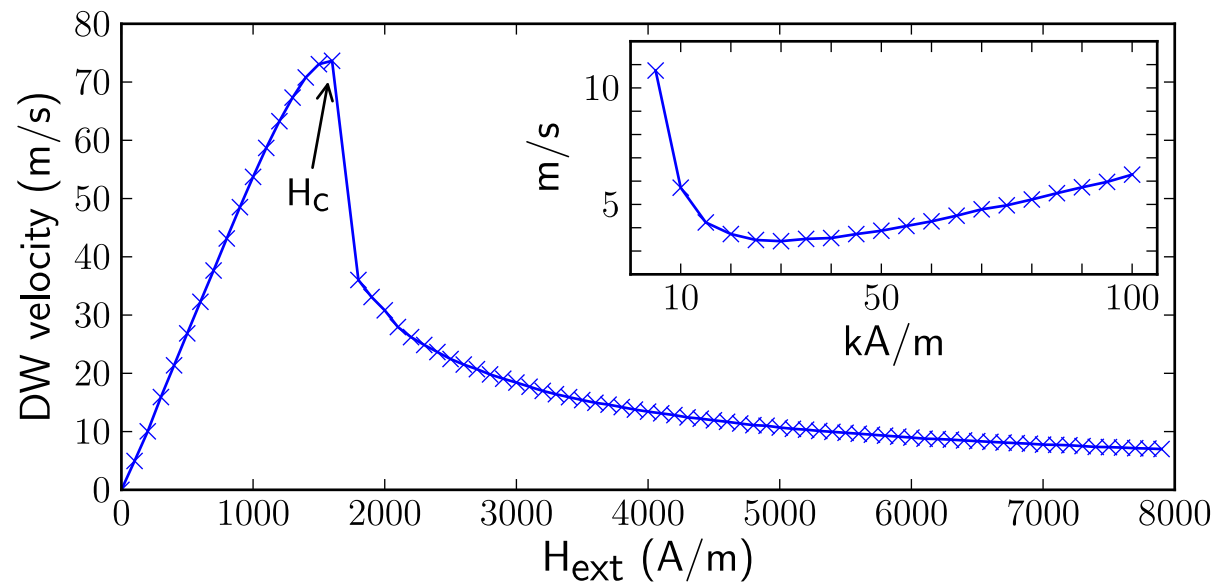

Figure 8. Domain wall velocity as a function of $\mathrm{H}_{\mathrm{ext}}$. The separation into two regimes, one above and one below the Walker breakdown, is clearly distinguishable. The inset shows the increase of the velocity for very strong fields $\gg H_{c}$ (note the different scale).

result, the system needs a certain applied field strength to depin the domain wall from this location and cause the domain wall to move. In this section we investigate how the edge roughness affects the domain wall motion (section 4.1), the domain wall velocity (section 4.2) and the depinning field (section 4.3).

\subsection{Domain wall motion}

We discuss the effect of roughness on domain wall motion for applied fields $H$ below (section 4.1.1) and above (section 4.1.2) the Walker breakdown field $\mathrm{H}_{\mathrm{c}}$ separately.

4.1.1. Influence of the roughness below the Walker breakdown Figure 9 (a) shows some typical domain wall trajectories in nanowires with different strengths of edge roughness in a fixed external field $H=0.6 \mathrm{kA} / \mathrm{m}$. One additional trajectory for a different field strength $H=0.5 \mathrm{kA} / \mathrm{m}$ (dotted curve) was included to illustrate dynamic pinning. The roughness correlation length is fixed at $c=4 \mathrm{~nm}$ whereas the roughness magnitude varies between $d=0 \mathrm{~nm}$ (smooth wire) and $d=0.06 \mathrm{~nm}$; for clarity, the curves for some intermediate values of $d$ have been omitted in the figure. The same randomisation seed was used in all cases so that the shape of the edge distortions is the same and only the amplitude $d$ varies. The particular edge roughness profile used is displayed at the top of the figure: we see the distortions introduced by the roughness functions $f(x)$ and $g(x)$ as introduced in section 2.3. For visibility, the vertical extents of the distortions $\mathrm{f}(\mathrm{x})$ and $\mathrm{g}(\mathrm{x})$ are scaled up in this plot. As mentioned previously, we plot the domain wall position $x$ along the horizontal axis. This allows us to compare the domain wall trajectories with the roughness profile that the domain wall centre is located in.

The trajectory in the smooth wire (dashed curve) shows the same characteristics as the ones discussed in section 3 : it takes the domain wall $\approx 3$ ns to reach its full speed, during which time the magnetisation angle $\phi$ increases and approaches its asymptotic 

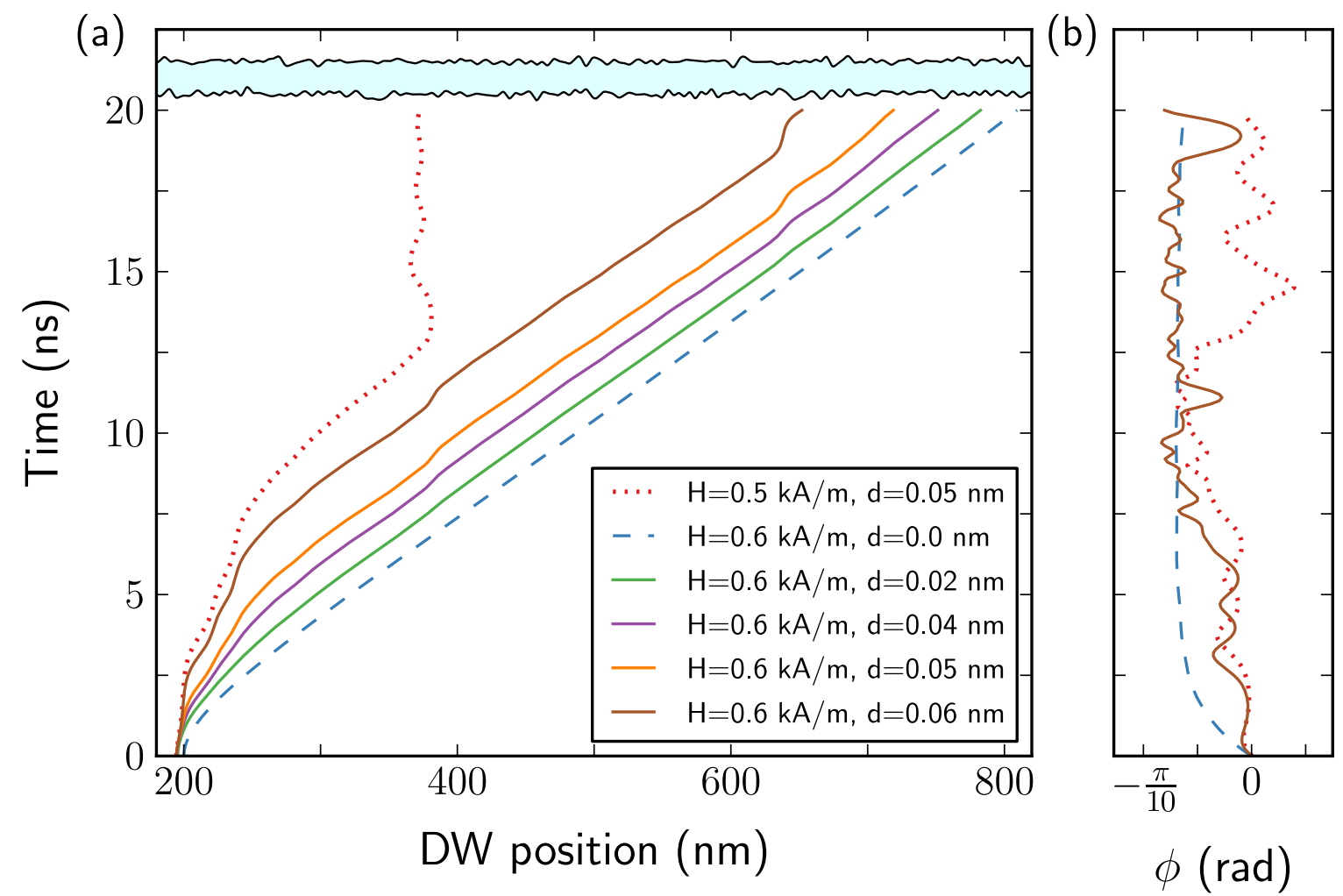

Figure 9. (Color online) (a) Sample trajectories in a constant external field $\mathrm{H}_{\text {ext }}=0.6 \mathrm{kA} / \mathrm{m}<\mathrm{H}_{\mathrm{c}}$ for varying roughness magnitudes $d$. The roughness correlation length is fixed at $c=4 \mathrm{~nm}$. One additional trajectory for a different field strength $H=0.5 \mathrm{kA} / \mathrm{m}$ (dotted curve) was included to illustrate dynamic pinning. The particular edge roughness profiles used in these simulations is shown as an inset towards the top: the roughness has been scaled up along the vertical axis to make the profile visible more easily. (b) Time evolution of the magnetisation angle $\phi$ for selected trajectories $d=0 \mathrm{~nm}$ (dashed) and $d=0.06 \mathrm{~nm}$ (solid) at $\mathrm{H}_{\text {ext }}=0.6 \mathrm{kA} / \mathrm{m}$ and $d=0.05 \mathrm{~nm}$ at $\mathrm{H}_{\text {ext }}=0.5 \mathrm{kA} / \mathrm{m}$ (dotted).

value as can be seen in figure 9 (b). Once this is reached so that the surface charges on the sides of the wire balance the torque which the external field exerts on the magnetisation inside the DW, the DW moves along the nanowire with constant velocity.

The trajectories for non-zero roughness show two marked differences in comparison to the smooth system. Firstly, it takes a longer time for the domain wall to depin and for the angle $\phi$ to approach its asymptotic value. The effect is the stronger the greater the roughness magnitude $d$ as shown by solid lines in figure 9 (a). For $d=0.02 \mathrm{~nm}$ the effect is small and the DW reaches its full speed after $\approx 4$ ns. For $d=0.06 \mathrm{~nm}$ this initial phase takes $\approx 8 \mathrm{~ns}$ - almost triple the time of the domain wall in the smooth nanowire $(d=0 \mathrm{~nm})$. For $d=0.06 \mathrm{~nm}$, we can see from Figures 9 (a) and (b) that the DW reacts to the edge distortions between the $x=200 \mathrm{~nm}$ and $x=250 \mathrm{~nm}$ during this initial transient.

The second difference between the trajectories in the smooth and the rough 
nanowires is that we observe local decelerations of the DW during the motion in the presence of edge roughness. In the examples shown in figure 9 (a) these manifest themselves as bumps in the curves and occur at $x \approx 380 \mathrm{~nm}$ and $x \approx 640 \mathrm{~nm}$. For the lower roughness magnitudes $(d=0.02 \mathrm{~nm}, 0.04 \mathrm{~nm})$ these are hardly noticeable, whereas for $d=0.05 \mathrm{~nm}$ and $0.06 \mathrm{~nm}$ they become visible. However, for the largest part of the motion the DW couples very weakly to the edge distortions and the curve is effectively straight (corresponding to constant velocity) with the velocity being virtually the same as in the smooth nanowire in these sections between the decelerations. If the driving field is too weak then the DW can get dynamically pinned during one of these decelerations. This is illustrated by the dotted curve in figure 9 (a).

In order to gain a better understanding of these two phenomena it is helpful to look at the magnetisation angle $\phi$ inside the DW. Figure 9 (b) shows the time evolution of $\phi$ as the DW progresses along the nanowire. In the smooth system (dashed curve) $\phi$ gradually increases from zero until it reaches its asymptotic value and then remains constant. For $d=0.06 \mathrm{~nm}$ (solid line) the angle $\phi$ shows a much more erratic behaviour as a function of time as the magnetisation reacts to the edge distortions in an attempt to minimise the surface charges at the sides. The value of $\phi$ for the domain wall in the wire with $d=0.06 \mathrm{~nm}$ approaches the asymptotic value of $\phi$ in the smooth system at $t \approx 8$ ns. For larger values of $t$ the angle $\phi$ shows small, apparently random deviations from this value. There are two exceptions at $t \approx 11 \mathrm{~ns}$ and $t \approx 19$ ns where $|\phi|$ gets close to zero, resulting in two larger spikes in the dashed curve. These correspond to the two local decelerations visible in figure 9 (a).

The dotted curve shows the time evolution of $\phi$ in the slightly weaker field $H=0.5 \mathrm{kA} / \mathrm{m}$, corresponding to the dotted trajectory in figure 9 (a). The way in which $\phi$ reacts to the edge distortions during the first half of the simulation is virtually identical to $H=0.6 \mathrm{kA} / \mathrm{m}$, except that the changes happen more slowly due to the lower driving field. Thus the first half of the dotted curve in figure $9(\mathrm{~b})$, before $t=12 \mathrm{~ns}$, is just a slightly vertically stretched version of the solid curve. The DW reaches the pinning site $x \approx 380 \mathrm{~nm}$ at $t \approx 11 \mathrm{~ns}$ in the stronger field (solid curve) and at $t \approx 13 \mathrm{~ns}$ in the weaker field (dotted curve). In the first case the field is strong enough to push the DW past the pinning site, which only results in a small spike towards zero of the angle. For the weaker field, on the other hand, the DW gets pinned and $\phi$ slowly relaxes back into the zero-position where the magnetisation points along the nanowire axis.

4.1.2. Influence of the roughness above the Walker breakdown Figure 10 (a) shows three trajectories of domain walls in an external field $H=5.75 \mathrm{kA} / \mathrm{m}>\mathrm{H}_{\mathrm{c}}$. The roughness correlation length $c=4 \mathrm{~nm}$ is fixed and only the roughness magnitude $d$ varies between $0.3 \mathrm{~nm}$ and $0.5 \mathrm{~nm}$. All trajectories show the oscillatory motion typical of fields $H>\mathrm{H}_{\mathrm{c}}$. In the absence of roughness (i.e. $d=0.0 \mathrm{~nm}$ ), all three curves would coincide.

The trajectory for the smallest roughness magnitude $d=0.3 \mathrm{~nm}$ (dashed red curve) is the most regular of the three and still quite similar to the trajectory in the smooth wire (not shown to avoid clutter in the plot). For the next larger value of roughness 

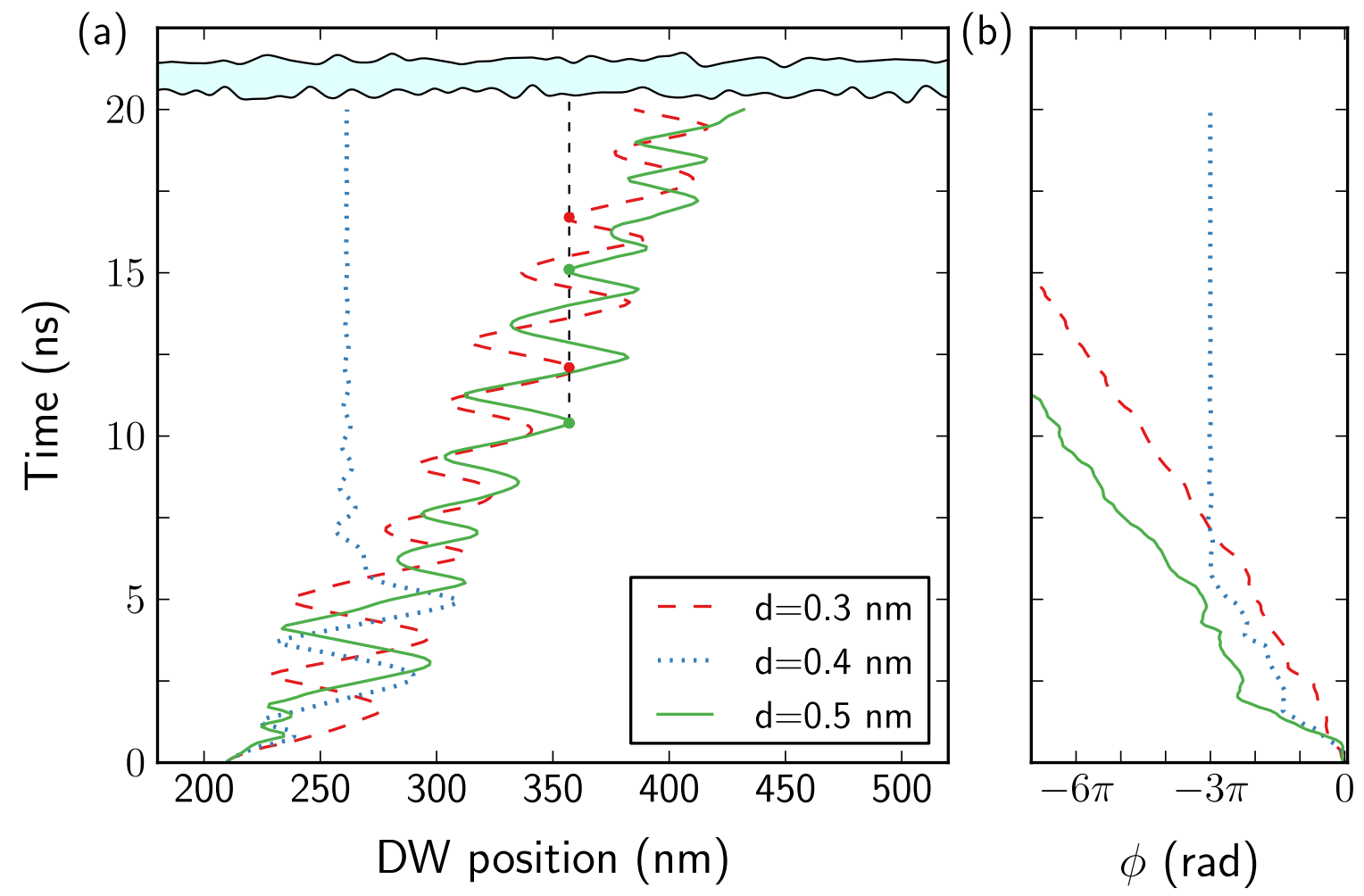

Figure 10. (Color online) (a) Sample trajectories in a constant external field $\mathrm{H}_{\text {ext }}=5.75 \mathrm{kA} / \mathrm{m}$ for varying roughness magnitudes $d$. The roughness correlation length is fixed at $c=4 \mathrm{~nm}$. The inset at the top shows the profile of the nanowire with roughness as in figure 9. (b) Time evolution of the magnetisation angle $\phi$ for these trajectories.

magnitude $d=0.4 \mathrm{~nm}$ (dotted blue curve) the edge distortions around $x=230 \mathrm{~nm}$ result in one short cycle with two quick direction reversals during the first 2 ns. Subsequently, the DW performs two larger position oscillation cycles before it gets caught by a pinning site at $x=261 \mathrm{~nm}$ and relaxes into a metastable state at this location. For the largest value shown in the plot, $d=0.5 \mathrm{~nm}$ (solid green curve), the domain wall reacts even more strongly to the edge roughness around $x=230 \mathrm{~nm}$, this time performing four quick direction reversals during the first $2 \mathrm{~ns}$, before it continues in a fashion similar to the dashed curve $(d=0.3 \mathrm{~nm})$.

The $x$-positions where the domain wall reverses direction during the oscillations in each of the trajectories are not arbitrarily distributed along the nanowire. Rather, the direction reversals nearly always fall into a constriction of the wire. The dashed black vertical line at $x=357 \mathrm{~nm}$ indicates one example where four direction reversals at different time steps in two trajectories (marked with green and red dots) fall into the same constriction. The same applies to virtually all other cycles in the three curves. This shows that the DW couples rather strongly to the edge distortions at the left and right turning points of the domain wall position cycles. Thus individual cycles can be lengthened or shortened as the DW reacts to the roughness, which accounts for the 
alterations in the trajectories visible for higher roughness strengths. By contrast, the edge distortions do not have a noticeable influence on the motion in the middle of a cycle.

For $d=0.4 \mathrm{~nm}$ the DW gets dynamically pinned at $x=261 \mathrm{~nm}$, whereas pinning does not occur for the smaller roughness magnitude $d=0.3 \mathrm{~nm}$ nor the larger one $d=0.5 \mathrm{~nm}$. Similarly, other simulation runs at a fixed roughness magnitude $d$ have shown that it is possible for the DW to get dynamically pinned in an external field of a certain strength while no pinning occurs for higher or lower fields.

We explain these observations by studying how the magnetisation angle $\phi$ in the domain wall couples to the edge distortions in the nanowire in the next section.

4.1.3. Discussion Dynamic pinning occurs where the total energy of the domain wall can be reduced by moving it into the pinning position. The domain wall carries a certain exchange and anisotropy energy which grow proportionally with the length (extension in $y$-direction) of the domain wall. The domain wall energy can thus be reduced if it moves to locations where the edge distortions on both sides of the nanowire collude to form a constriction, and thus reduce the length of the domain wall. For example, the domain wall for $d=0.4 \mathrm{~nm}$ that is shown as a dotted line in figure 10 (a) is dynamically pinned at $x \approx 261 \mathrm{~nm}$, and we see a constriction in the roughness profile which is shown as an inset in the top of the figure.

Above the Walker breakdown, the angle $\phi$ keeps growing, which reflects the rotation of the magnetisation in the $x-y$-plane during the oscillatory motion of the domain wall; the displacement of the domain wall and the change in $\phi$ are coupled as described in section 3 and visible in figure 6 . For $\phi=n \pi$ with $n=0, \pm 1, \pm 2, \ldots$, the domain wall magnetisation points along the wire and there are no surface charges along the edges associated with the domain wall, so the demagnetisation energy of the domain wall is minimal. Conversely, for $\phi=\left(n+\frac{1}{2}\right) \pi$ the magnetisation points in $\pm y$ direction, and this maximises the demagnetisation energy associated with the domain wall. Between those extrema the energy varies continuously. The increase in demagnetisation energy due to $\phi$ changing from, say, 0 to $\frac{\pi}{2}$ is large in comparison to the energy fluctuations caused by the edge roughness, such as the decrease in energy due to a reduced domain wall length in a constriction.

Therefore the domain wall only interacts with the roughness where the energy reduction caused by a constriction is of a magnitude comparable to the change in the demagnetisation energy associated with a small displacement of the domain wall, or equivalently a small change in $\phi$. The response of the demagnetisation energy to a change in $\phi$ is smallest around extrema, i.e. around $\phi=n \pi$ (minima) and $\phi=\left(n+\frac{1}{2}\right) \pi$ (maxima). For intermediate values of $\phi$, the energy change due to roughness is insignificant in comparison to the dominating demagnetisation energy change.

This interpretation explains why the turning points of the positions of the domain wall tend to coincide with constrictions in the roughness pattern: at the left-hand turning points we have $\phi=n \pi$ and at the right-hand turning points $\phi=\left(n+\frac{1}{2}\right) \pi$, so that 
the DW can couple to the edge distortions at these points in the motion. Figure 10 (a) shows selected turning points of the oscillating domain wall position and illustrates how these align with the effective constrictions in the roughness of the wire as indicated by the vertical dashed black line.

The same argument suggests that dynamic pinning above the Walker breakdown can only occur for $\phi \approx n \pi$ and $\phi \approx\left(n+\frac{1}{2}\right) \pi$. In our simulations, we have only observed dynamic pinning where $\phi \approx n \pi$. An example for this dynamic pinning above the Walker breakdown is shown in figure 10 (a) for $d=0.4 \mathrm{~nm}$ as a dotted line. The domain wall shows oscillatory motion up to $t \approx 5 \mathrm{~ns}$, and subsequently gets pinned at $t \approx 7 \mathrm{~ns}$. The corresponding dotted line in figure $10(\mathrm{~b})$ shows that $\phi=-3 \pi$, i.e. $\sin (\phi)=0$, when the domain wall reaches the pinning site. The requirement that $\phi$ must be close to $n \pi$ or $\left(n+\frac{1}{2}\right) \pi$ for dynamic pinning to occur above the Walker breakdown explains why the domain wall $d=0.4 \mathrm{~nm}$ in figure 10 (a) can pass through the pinning site $x \approx 260 \mathrm{~nm}$ repeatedly without being pinned (three times for $t<5 \mathrm{~ns}$ ).

Below the Walker breakdown, there is competition between the external field that drives the domain wall forward and the effective potential that the domain wall experiences due to the roughness. If the roughness exhibits a constriction, this reduces the domain wall length and energy. The constriction can be interpreted as a pinning potential well that the domain wall experiences if we use a model where the domain wall is a particle that experiences a spatially varying potential energy. It depends on the depth and width of this potential well whether the applied field can push the domain wall through it or whether pinning takes place. An increasing external field reduces the pinning strength. This is in line with our observations that pinning becomes less likely for larger fields $H$ below the critical Walker breakdown field.

We hypothesise that in addition to the length (in $y$-direction) of a constriction in the roughness profile, it is also its width (in $x$-direction) which contributes to how effective a constriction is as a dynamic pinning centre: presumably constrictions of width comparable to the domain wall width are most effective, but the detailed shape of the constriction is likely to be important, too.

The discussion above shows that in the steady and oscillatory regimes the pinning process, although guided by the same underlying principles, leads to rather different phenomena. For $H<\mathrm{H}_{\mathrm{c}}$ the probability of the DW being pinned decreases with increasing external field $H$ since higher fields lead to a larger asymptotic value of $\phi$, whereas for $H>\mathrm{H}_{\mathrm{c}}$ there is no simple relationship between the strength of the field and the pinning probability since the latter depends on the intricate interaction of the constantly precessing magnetisation and the edge distortions. Our simulation results have shown that pinning above the Walker breakdown occurs in the whole range up to the highest simulated fields $(10 \mathrm{kA} / \mathrm{m})$ and appears to be more common for higher fields than for fields just above $\mathrm{H}_{\mathrm{c}}$. This seemingly counter-intuitive observation can be explained by the fact that the oscillations of the DW position are much shorter for large fields $H$ than for small fields. For large fields, the domain wall position trajectory overlaps with itself (see the quickly oscillating curves in figures 5 (a) and 10 (a)). Thus 
the DW passes the same location in the nanowire more often, with different angles. This makes it more likely to reach a pinning site with $\phi=n \pi$ or $\phi=\left(n+\frac{1}{2}\right) \pi$ and thus increases the pinning probability.

\subsection{Influence of the roughness on the domain wall velocity}

The domain wall trajectories in figure 10 (a) show that, although individual oscillation cycles can be significantly altered at different roughness levels $d$, the changes mostly average out over time. Thus the mean velocities are quite similar for all roughness strengths. This is in line with the observation which we made earlier for $H<\mathrm{H}_{\mathrm{c}}$ in figure 9 (a), where the asymptotic velocities are also virtually identical for all trajectories. In this section we study the influence of the roughness on the domain wall velocity in more detail.

As discussed in section 2.4, there are two distinct ways of computing the velocity: (i) the mean velocity, which uses the distance travelled during the simulated time based on the first and last point of the trajectory; (ii) the asymptotic velocity, which attempts to estimate the asymptotic velocity by disregarding the depinning process and, for fields above the Walker breakdown, removes artefacts due to incomplete oscillation cycles at the end of the simulation. We discuss both methods, putting our main emphasis on the second one.

Figure 11 (a) shows plots of the asymptotic domain wall velocity (section 2.4.2) as a function of the applied field for various roughness magnitudes $d$. Figure 11 (b) shows the corresponding mean velocity (section 2.4.1). The correlation length of the roughness is $c=6.0 \mathrm{~nm}$ for all data shown while the roughness amplitude varies between $d=0.0 \mathrm{~nm}$ (smooth wire) and $d=0.09 \mathrm{~nm}$ in steps of $0.01 \mathrm{~nm}$. The same randomisation seed was used to produce the roughness profile in all cases so that increasing $d$ does not change the shape of the edge roughness but only increases the vertical size of the distortions. For each value of $d$ the external field was increased from $0 \mathrm{~A} / \mathrm{m}$ up to $3000 \mathrm{~A} / \mathrm{m}$ in steps of $100 \mathrm{~A} / \mathrm{m}$ and a simulation was run for each applied field value in order to compute the DW velocities. In a second phase the external field interval containing the depinning field was discretised in finer steps of $10 \mathrm{~A} / \mathrm{m}$ to obtain a better resolution.

We define the depinning field $\mathrm{H}_{\text {depin }}$ as the smallest field that is just strong enough to drive the DW away from its original position into which the system has been relaxed during the first stage of the simulation, irrespective of whether the DW gets pinned at a later stage or not. The depinning field is a function of the correlation length $c$ and roughness magnitude $d$ that define the edge roughness: $\mathrm{H}_{\text {depin }}=\mathrm{H}_{\text {depin }}(c, d)$. For the discussion of figure 11, $c$ is kept fixed at $6 \mathrm{~nm}$.

The line for $d=0 \mathrm{~nm}$ in figure 11 corresponds to a nanowire without roughness, and there is no depinning field defined: any finite applied field will result in domain wall motion. For increasing roughness magnitude we see that the domain wall remains pinned in its original position (corresponding to zero velocity) up to the critical depinning field, which increases with increasing $d$. Once the external field $H$ exceeds $\mathrm{H}_{\text {depin }}$ for a given 
(a)

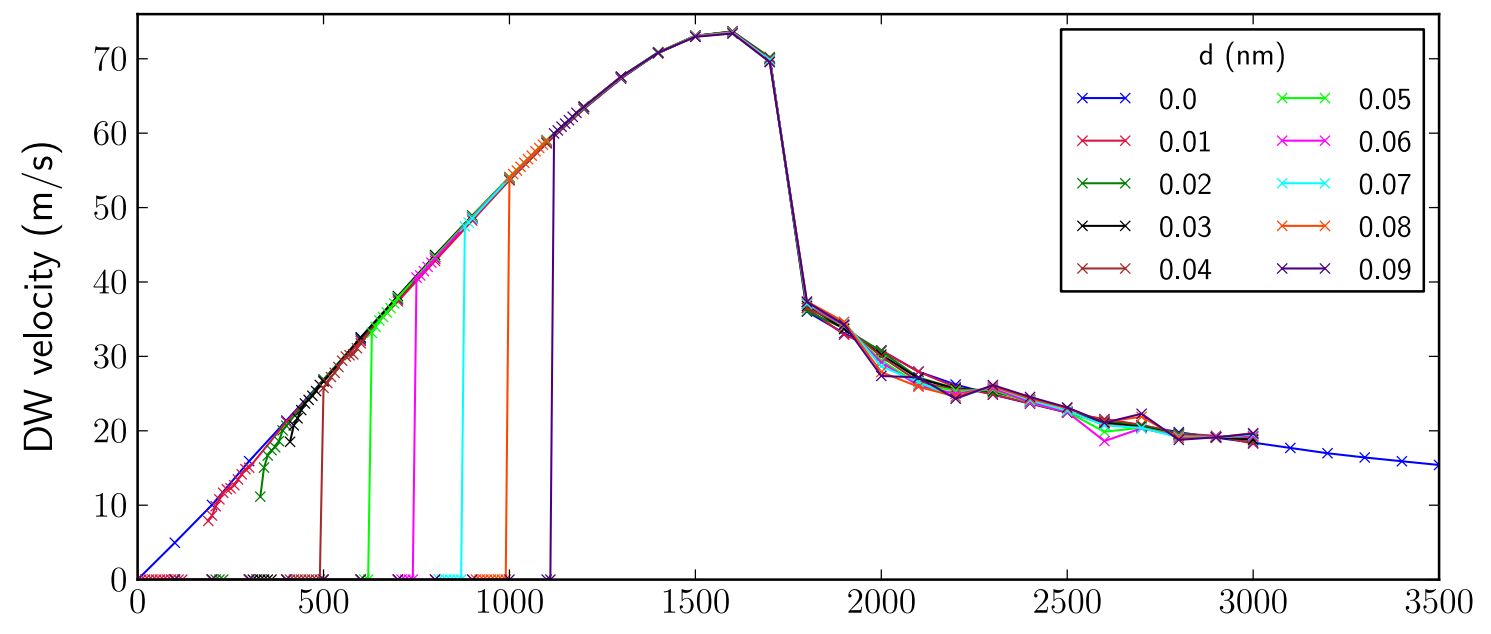

(b)

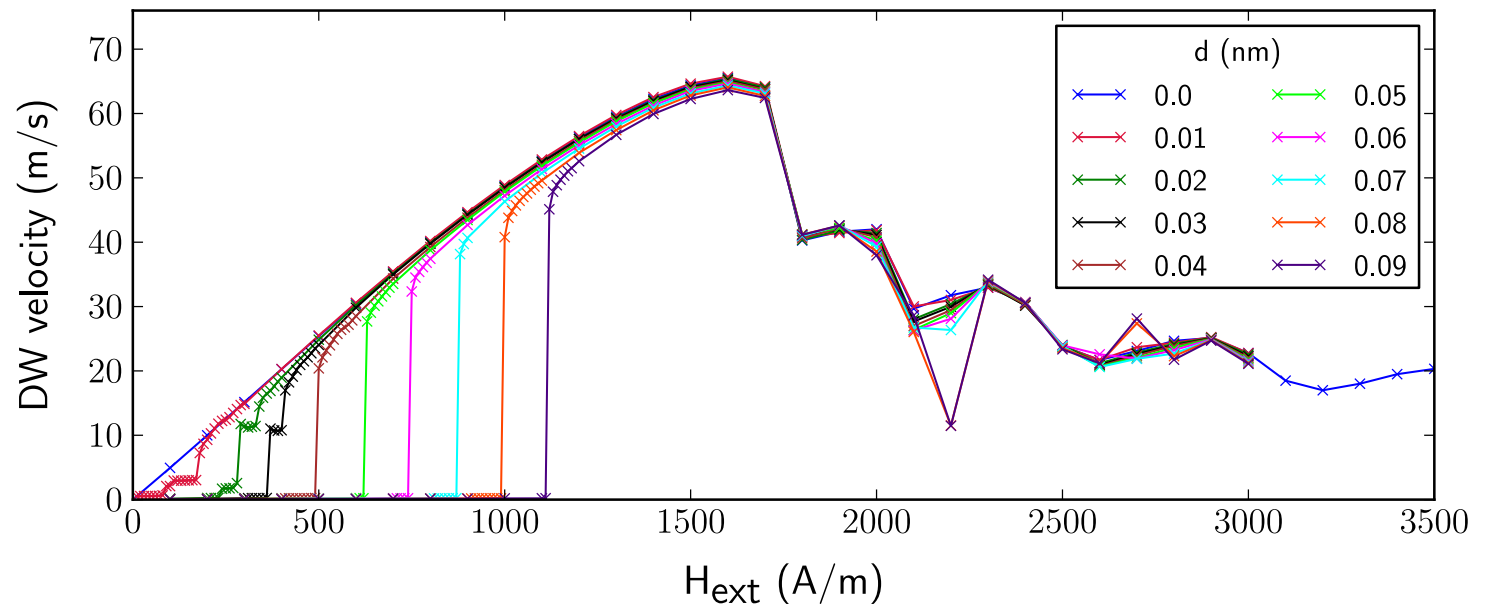

Figure 11. (Color online) (a) Asymptotic domain wall velocity, computed using the method described in section 2.4.2, as a function of applied field $H$. Each line corresponds to one roughness magnitude $d$. (b) Corresponding mean domain wall velocity, computed using method described in section 2.4.1.

roughness magnitude $d$, the DW starts moving.

For a couple of small values of $d$, such as $d=0.02 \mathrm{~nm}$ or $0.03 \mathrm{~nm}$, the DW mean velocity appears to increase 'in leaps' (figure $11(\mathrm{~b})$ ), which is due to the DW being pinned dynamically for small fields so that the mean velocity is reduced. This is not visible in the asymptotic velocity (figure $11(\mathrm{a})$ ) where these data points have been removed as in these cases the asymptotic velocity is not defined (see section 2.4.3). However, for larger $d$ such as $d=0.04-0.09 \mathrm{~nm}$ the asymptotic velocity appears, on the scale of this graph, to jump immediately from zero to the velocity of the smooth system. For stronger applied fields up to the critical Walker field $\mathrm{H}_{\mathrm{c}} \approx 1.7 \mathrm{kA} / \mathrm{m}$ the asymptotic DW velocities are virtually identical for all roughness strenghts. Above the Walker breakdown there is some slight variation, but the discrepancies are small $(\approx 10 \%$ for the largest deviations, e.g. for $H=2.0 \mathrm{kA} / \mathrm{m}$ ).

The plot of the domain wall mean velocities in figure $11(\mathrm{~b})$ shows that there is 
a somewhat gradual increase in the mean velocity once $H_{\text {ext }}$ exceeds $H_{\text {depin }}$ and that up to the Walker field $\mathrm{H}_{\mathrm{c}}$ the DW velocity in rough nanowires always stays below the value of the smooth wire. The larger the roughness magnitude $d$, the further does the mean velocity stay below the domain wall velocity of the smooth wire. It appears as if larger roughness reduces the velocity, but the comparison with the asymptotic velocities (figure 11 (a)) reveals that it is the initial depinning process that gets increasingly slower with increasing roughness magnitude $d$ (see section 4.1.1). As the mean velocity is a time average of the domain wall velocity, this initial slowdown is visible. We note that the reduction of the mean velocity due to the depinning will decrease if the domain wall motion is simulated for longer periods of time. Unless we want to study the effect of the depinning process on the mean velocity, we prefer the asymptotic velocity as an observable because it is independent of the simulated time.

Above the critical Walker field, the mean velocity curves in figure 11 (b) exhibit a seemingly erratic behaviour. Even the one for the smooth system $(d=0.0 \mathrm{~nm})$ shows a kind of undulation which the other curves more or less follow. To illustrate this more clearly, the curve for the smooth system has been extended up to $H=3.5 \mathrm{kA} / \mathrm{m}$. This behaviour can be explained as follows. Since the DW motion is oscillatory, the end point of the trajectory can vary significantly depending on where last oscillation is cut off when the simulation exits. With the exit time of the simulation remaining constant at $20 \mathrm{~ns}$ but the oscillations becoming shorter for increasing $\mathrm{H}_{\text {ext }}$, the cut-off point varies significantly and also jumps backward and forward, leading to the undulating values of the velocity within each curve in figure $11(\mathrm{~b})$. The two outliers for $H=2.2 \mathrm{kA} / \mathrm{m}$ indicate that the DW was dynamically pinned for these two roughness levels, which affects the mean velocity but is filtered out by our method to compute the asymptotic velocity.

The observation that the asymptotic velocity in the presence of roughness coincides with the domain wall velocity without roughness is in agreement with the data and discussion in sections 4.1.1 and 4.1.2: Figures 9 (a) and 10 (a) illustrate that even though there are perturbations in the trajectories - local decelerations below $\mathrm{H}_{\mathrm{c}}$ and alterations of the oscillations above $\mathrm{H}_{\mathrm{c}}$ - the asymptotic velocities are effectively the same as in the smooth nanowire even for higher roughness amplitudes.

Figure 11 shows that the critical Walker breakdown field is the same for all roughness strengths, which was observed in all other performed simulation runs as well. This is in contrast to a similar study of in-plane domain walls (Min et al. 2010), where an increase of the critical field (combined with a slightly decreased peak DW velocity) was observed for higher roughness magnitudes.

We have discussed two different ways to analyze data from the time dependent simulations to compute (i) the mean velocity, and (ii) the asymptotic velocity. Depending on the experimental context, either entity may be of interest: for domain wall motion in very short nanowires, inclusion of the depinning time as in the mean velocity calculation may be desired. If the simulation is meant to simulate a system where the depinning time is irrelevant (because the wire is very long) but matters in 


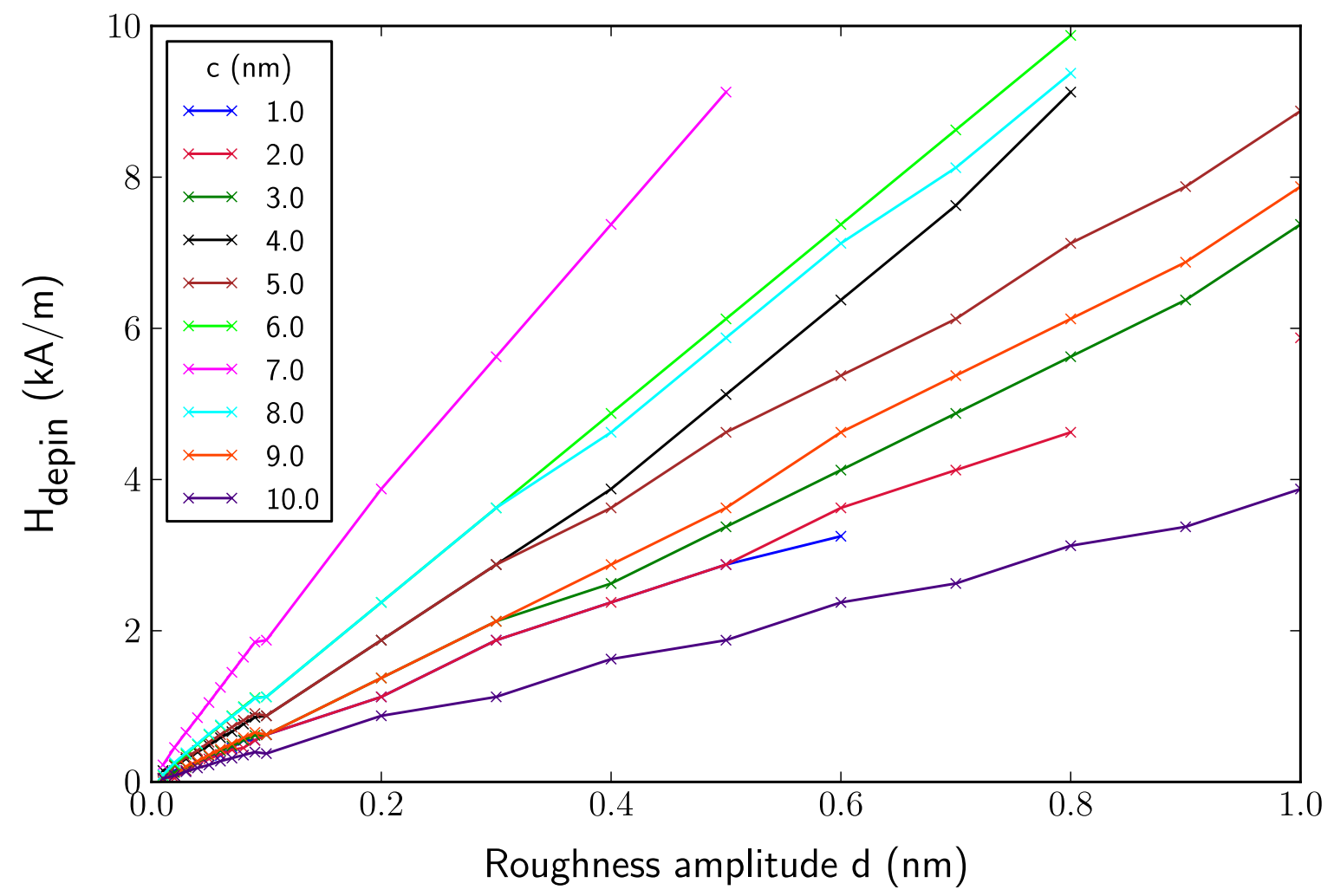

Figure 12. (Color online) Depinning field $\mathrm{H}_{\text {depin }}$ as a function of the roughness amplitude $d$, for different correlation lengths $c$.

the simulation (because the simulated wire is not so long), then the asymptotic velocity may be a better measure.

\subsection{Influence of the roughness on the depinning field}

In the study of figure 11, where the roughness correlation length is $c=6 \mathrm{~nm}$, we have noted that the depinning field $\mathrm{H}_{\text {depin }}(c, d)$ increases as a function of roughness magnitude $d$. In this section, we analyse this dependence quantitatively for varying correlation lengths $c$. The corresponding simulation results are gathered in figure 12 . Each of the curves displays the dependence of the depinning field $\mathrm{H}_{\text {depin }}$ on the roughness magnitude $d$ for a fixed roughness correlation length $c$. The data shows that the depinning field has an approximately linear dependence on the roughness magnitude $d$ for all values of $c$. We denote the constant of proportionality in this linear relationship by $\beta_{c}$, so that $\mathrm{H}_{\text {depin }}(c, d)=\beta_{c} \cdot d$. Thus $\beta_{c}$ is a measure of the effective pinning strength of the edge roughness for a given roughness correlation length $c$, and given by the slope of the lines in figure 12. We use a least-squares fit to determine the value of $\beta_{c}$ for each line, omitting the data points for $0.01 \leq d \leq 0.09$ to avoid a bias.

Figure 13 shows how $\beta_{c}$ varies as a function of $c$. The data points marked with a cross and connected by a solid line show the slopes of the ten lines that are plotted in 


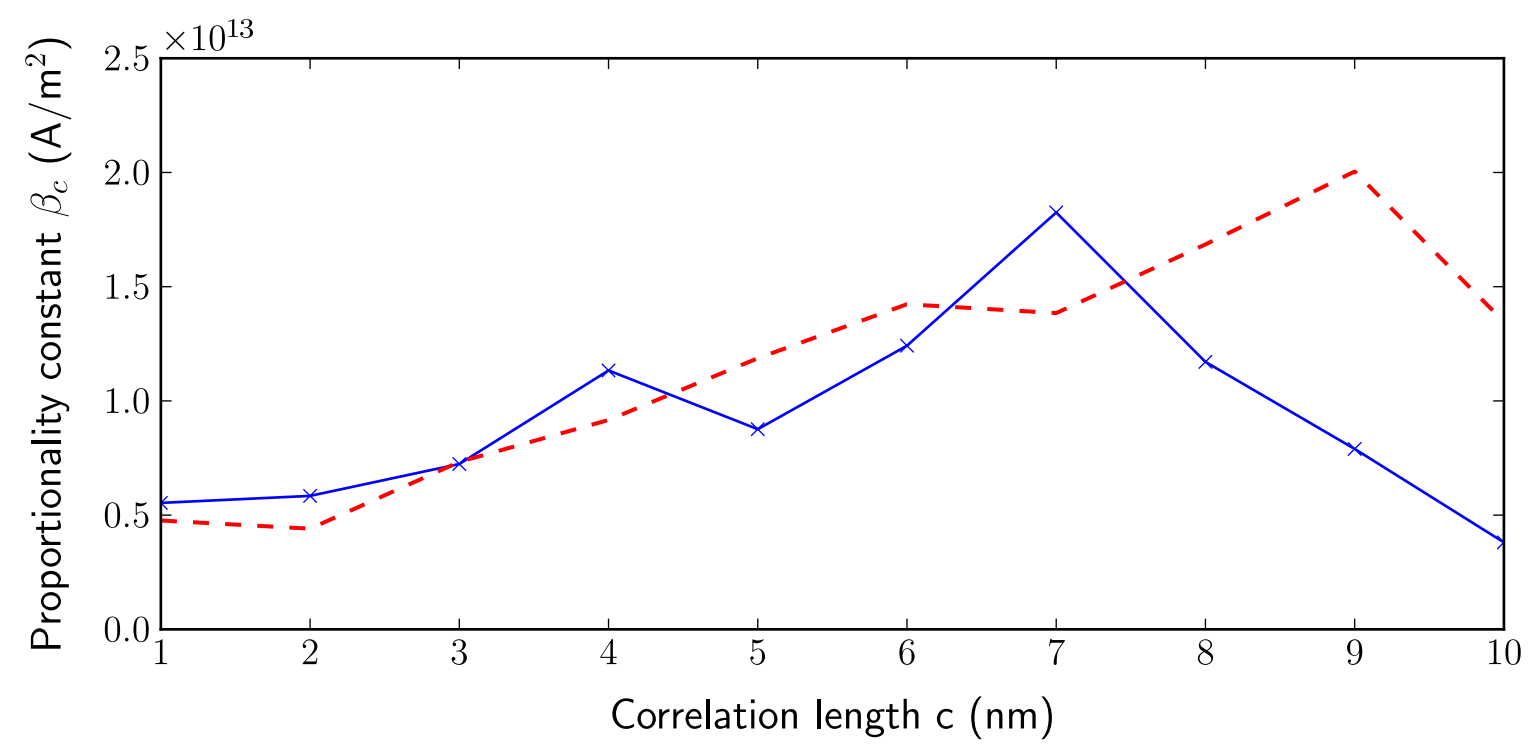

Figure 13. Proportionality constants $\beta_{c}$ describing the dependence of the depinning field on the roughness level $d$ for each correlation length $c$. The solid line corresponds to figure 12; the dashed line represents values averaged over four runs with different roughness shapes.

figure 12. The dashed line shows corresponding results that have been averaged over four different sets of simulation runs (i.e. using four different random seeds for the domain wall roughness functions, and then repeating all simulations required to obtain the $\beta_{c}$ 's). Both curves show low values for small and large $c$ and a maximum between at $c=7 \mathrm{~nm}$ (solid line) and $c=9 \mathrm{~nm}$.

The largest influence of the roughness on the DW motion is expected if the characteristic length scale of the edge distortions is of the same order as the domain wall width: for very small correlation lengths $c$ the roughness is at a scale too small to be noticed by the DW, whereas for very large values of $c$ the wire edge appears locally flat to the DW. The characteristic domain wall width is taken as $\pi \sqrt{A / K_{1}}$ (Lilley 1950, Kronmüller \& Fähnle 2003), which is $18.4 \mathrm{~nm}$ for our material parameters. The effective roughness length scale for a given parameter $c$ is on the order of $2.76 c$ as shown in section 2.3. Matching of the effective roughness length scale with the domain wall width should thus occur where $c=\pi \sqrt{A / K_{1}} / 2.76 \approx 6.7 \mathrm{~nm}$. This is compatible with the data shown in figure 13 where the most effective pinning is found for $c$ in the range $7 \mathrm{~nm}$ to $9 \mathrm{~nm}$.

Finally, focusing on the range of values which $\beta_{c}$ takes for different roughness correlation lengths $c$, we note that by matching the effective roughness length scale with the domain wall width, the pinning effect can be increased significantly (approximately a factor of 5 for the data shown here). 


\section{Summary}

We study the dynamics of field-driven domain walls in perpendicular magnetic anisotropy (PMA) nanowires with added edge roughness. We use a finite-element based roughness model which allows systematic exploration of the roughness configuration space using two parameters: (i) the roughness length scale $c$ and (ii) the roughness magnitude $d$ (section 2.3).

The dynamics of a domain wall moving in a smooth nanowire is studied in order to have a reference point for the rough systems. The typical Walker breakdown is observed, with the domain wall showing steady motion below the critical Walker field $\mathrm{H}_{\mathrm{c}}$ and oscillatory motion above $\mathrm{H}_{\mathrm{c}}$. In the smooth system the DW dynamics can be understood in terms of the precession of the magnetisation angle $\phi$ inside the domain wall.

In the nanowire with edge roughness, the Walker breakdown is observed at the same critical applied field $\mathrm{H}_{\mathrm{c}}$ as in the smooth system. Whereas in the smooth system the DW moves for any non-zero applied field, with added edge roughness the domain wall remains pinned up to a critical depinning field $\mathrm{H}_{\text {depin }}$ which increases with increasing roughness magnitude $d$. For fields $H>\mathrm{H}_{\text {depin }}$ the roughness affects the DW trajectories. In the steady-state regime below the Walker breakdown the roughness leads to a significantly prolonged initial depinning process and introduces local decelerations in the DW motion, resulting in sporadic distortions of the trajectory which otherwise remains largely unchanged (figure $9(\mathrm{a})$ ). In the oscillatory regime the individual DW cycles can be markedly altered in size and shape (figure 10 (a)).

The presence of edge distortions can also lead to dynamic pinning during the DW motion. This is a process which is stochastic in nature. We find that dynamic pinning above the walker breakdown is only likely to happen when the magnetisation angle $\phi$ is aligned with, or perpendicular to, the long wire-axis, as these are the points in the trajectory where the domain wall couples most strongly to the roughness distortions.

We study the influence of the roughness on the domain wall velocity, and compute two different observables: the mean velocity and the asymptotic velocity. The results have shown that the mean velocity of the domain wall during the simulation can be significantly lower than in the smooth system (figure $11(\mathrm{~b})$ ). This slowdown occurs for fields slightly above the depinning field and is the result of the prolonged initial depinning process in the presence of rough edges (figure 9 (a)). However, the asymptotic DW velocity, which ignores the initial depinning, is essentially unaltered by the presence of roughness (figure $11(\mathrm{a})$ ), in spite of the noticeable influence of the roughness on the DW trajectories. In contrast to the mean velocity observable, the data obtained for the asymptotic velocity does not depend on the length of the simulated time.

Finally, we study the dependence of the depinning field on the roughness parameters in our model. We find that $\mathrm{H}_{\text {depin }}$ increases approximately linearly with the roughness amplitude $d$, which controls the vertical size of the edge distortions. We show that the effectiveness of pinning increases considerably if the width of the roughness peaks and troughs correlates with the domain wall width. 


\section{Acknowledgments}

The authors would like to thank Stuart Curtis for useful discussions and Peter Metaxas for very valuable comments and suggestions. The research leading to these results has received funding from the European Communitys Seventh Framework Programme (FP7/2007-2013) under Grant Agreement n233552 (DYNAMAG), and from the EPSRC (EP/E040063/1 and Doctoral Training Centre Grant EP/G03690X/1). Financial support by the Deutsche Forschungsgemeinschaft via SFB 668 as well as GK 1286 and from the Cluster of Excellence Nanospintronics funded by the Forschungs- und Wissenschaftsstiftung Hamburg is gratefully acknowledged.

\section{References}

Bocklage L, Krüger B, Matsuyama T, Bolte M, Merkt U, Pfannkuche D \& Meier G 2009 Phys. Rev. Lett. 103(19), 197204.

Burrowes C, Mihai A P, Ravelosona D, Kim J V, Chappert C, Vila L, Marty A, Samson Y, GarciaSanchez F, Buda-Prejbeanu L D, Tudosa I, Fullerton E E \& Attané J P 2009 Nature Physics 6(1), 17-21.

Fischbacher T, Franchin M, Bordignon G \& Fangohr H 2007 IEEE Transactions on Magnetics 43(6), 2896-2898.

Fischbacher T, Franchin M, Bordignon G, Knittel A \& Fangohr H 2009 Journal of Applied Physics 105(7), $07 \mathrm{D} 527$.

Hayashi M, Thomas L, Rettner C, Moriya R, Jiang X \& Parkin S S P 2006 Phys. Rev. Lett. 97(20), 207205.

Im M Y, Bocklage L, Fischer P \& Meier G 2009 Phys. Rev. Lett. 102(14), 147204.

Kim K J, Lee J C, Ahn S M, Lee K S, Lee C W, Cho Y J, Seo S, Shin K H, Choe S B \& Lee H W 2009 Nature 458(7239), 740-2.

Kläui M, Vaz C A F, Bland J A C, Wernsdorfer W, Faini G, Cambril E, Heyderman L J, Nolting F \& Rüdiger U 2005 Phys. Rev. Lett. 94(10), 106601.

Kronmüller H \& Fähnle M 2003 Micromagnetism and the Microstructure of Ferromagnetic Solids 1st edn Cambridge University Press. p 53.

Lilley B A 1950 Philos. Mag 41, 792.

Martinez E, Lopez-Diaz L, Torres L, Tristan C \& Alejos O 2007 Phys. Rev. B 75(17), 174409.

Metaxas P J, Jamet J P, Mougin A, Cormier M, Ferré J, Baltz V, Rodmacq B, Dieny B \& Stamps R L 2007 Phys. Rev. Lett. 99(21), 217208.

Min H, McMichael R D, Donahue M J, Miltat J \& Stiles M D 2010 Phys. Rev. Lett. 104(21), 217201.

Nakatani Y, Thiaville A \& Miltat J 2003 Nature materials 2(8), 521-3.

Nmag - a micromagnetic simulation environment 2007 http://nmag.soton.ac.uk/.

Parkin S, Hayashi M \& Thomas L 2008 Science 320, 190.

Press W H, Teukolsky S A, Vetterling W T \& Flannery B P 1992 Numerical Recipes in C 2nd edn Cambridge University Press.

Rodríguez-Rodríguez G, Menéndez J L, Hierro-Rodriguez A, Pérez-Junquera A, Montenegro N, Ravelosona D, Alameda J M \& Vélez M 2010 Journal of Physics D: Applied Physics 43(30), 305002.

Ryu J \& Lee H W 2009 Journal of Applied Physics 105(9), 093929.

Schryer N \& Walker L 1974 Journal of Applied Physics 45(12), 5406-5421.

Tanigawa H, Koyama T, Yamada G, Chiba D, Kasai S, Fukami S, Suzuki T, Ohshima N, Ishiwata N, Nakatani Y \& Ono T 2009 Applied Physics Express 2, 053002. 
Tatara G, Takayama T, Kohno H, Shibata J, Nakatani Y \& Fukuyama H 2006 Journal of the Physical Society of Japan $\mathbf{7 5 ( 6 ) , 0 6 4 7 0 8 .}$

Thiaville A, Nakatani Y, Miltat J \& Suzuki Y 2005 EPL (Europhysics Letters) 69(6), 990.

Vogel A, Wintz S, Gerhardt T, Bocklage L, Strache T, Im M Y, Fischer P, Fassbender J, McCord J \& Meier G 2011 Appl. Phys. Lett. 98, 202501.

Vogel A, Wintz S, Kimling J, Bolte M, Strache T, Fritzsche M, Im M Y, Fischer P, Meier G \& Fassbender J 2010 IEEE Transactions on Magnetics 46, 1708-1710. 